\title{
PERIODIC SOLUTIONS OF THE KORTEWEG-DE VRIES EQUATION DRIVEN BY WHITE NOISE
}

\author{
A. DE BOUARD ${ }^{1}$, A. DEBUSSCHE ${ }^{2}$, AND Y. TSUTSUMI $^{3}$
}

\author{
${ }^{1}$ CNRS et Université Paris-Sud, \\ UMR 8628, Bât. 425, Université de Paris-Sud, \\ 91405 ORSAY CEDEX, FRANCE \\ ${ }^{2}$ ENS de Cachan, Antenne de Bretagne, \\ Campus de Ker Lann, Av. R. Schuman, \\ 35170 BRUZ, FRANCE \\ ${ }^{3}$ Mathematical Institute, \\ Tohoku University, \\ SENDAI 980-8578, JAPAN
}

\begin{abstract}
We consider a Korteweg-de Vries equation perturbed by a noise term on a bounded interval with periodic boundary conditions. The noise is additive, white in time and "almost white in space". We get a local existence and uniqueness result for the solutions of this equation. In order to obtain the result, we use the precise regularity of the Brownian motion in Besov spaces, and the method which was introduced by J. Bourgain, but based here on Besov spaces.
\end{abstract}

\section{INTRODUCTION}

The Korteweg-de vries (KdV) equation, which models the propagation of unidirectional weakly nonlinear waves in an infinite channel, is an ideal model, and it is natural to consider perturbations of this model. In this direction, stochastic perturbations of this equation were introduced in [5], [12], [19] to model the propagation of weakly nonlinear waves in a noisy plasma.

Here, we consider as in [2], [3], a KdV equation with a stochastic perturbation which is Gaussian and of white noise type in time. Contrary to the previous works [2] and [3], we will set the equation on a bounded space interval with periodic boundary conditions. Although the derivation of the $\mathrm{KdV}$ equation is usually done with $x \in \mathbb{R}$, there is no reason to confine oneself to localized solutions. It is also well known that the $\mathrm{KdV}$ equation possesses spatially periodic traveling waves solutions. The study of the periodic boundary

\footnotetext{
Key words and phrases. Korteweg-de Vries equation, stochastic partial differential equations, white noise, Besov spaces.
} 
conditions case is also of importance when dealing with numerical computations, since these are necessarily performed on a bounded interval.

Our aim in the present paper is to study the Cauchy problem for a stochastic $\mathrm{KdV}$ equation with an additive noise as previously described, and which has spatial correlations "as rough" as our techniques allow, the aim being to stay as close as possible to the space-time white noise.

The equation is then written as

$$
\partial_{t} u+\partial_{x}^{3} u+u \partial_{x} u=\phi \frac{\partial^{2} B}{\partial t \partial x}
$$

where $u$ is a random process defined for $(t, x) \in \mathbb{R}^{+} \times \mathbb{T}$, $\mathbb{T}$ being a onedimensional torus, and $\phi$ is a bounded linear operator on $L^{2}(\mathbb{T})$ that will be described in more details later. Also, $B$ is a two parameter Brownian motion on $\mathbb{R}^{+} \times \mathbb{T}$, that is a zero mean Gaussian process whose correlation function is given by

$$
\mathbb{E}(B(t, x) B(s, y))=(t \wedge s)(x \wedge y)
$$

for $t, s \geq 0, x, y \in \mathbb{T}$.

Note that in the case where $\phi$ is defined by a kernel $k(x, y)$, then the correlation function of the noise is

$$
\mathbb{E}\left(\phi \frac{\partial^{2} B}{\partial t \partial x}(t, x) \phi \frac{\partial^{2} B}{\partial t \partial x}(s, y)\right)=c(x, y) \delta_{t-s}
$$

with $\delta$ the Dirac $\delta$-function and

$$
c(x, y)=\int_{\mathbb{T}} k(x, z) k(y, z) d z .
$$

In this formalism, the case $\phi=I d$ i.e. $c(x, y)=\delta(x-y)$ corresponds to the space-time white noise. This is the case we would like to treat. However, our result needs a slightly more restrictive assumption, and we are only able to treat a noise which is "almost" delta correlated in space.

Except in [2] and [3], equations of the type (1.1) have essentially been studied by using inverse scattering theory (only in the case where the noise is space independent) or by perturbation arguments near the integrable case (see [12], [16], [21], [22]).

A very large attention has been paid to the (deterministic) KdV equation on the real line (see [1], [4], [13], [18]) and improvements made on the regularity needed on the initial value to get local existence of solutions occurred step by step. On the opposite, for the periodic case, up to the famous work of Bourgain on the $\mathrm{KdV}$ equation (see [4]), existence results in $H^{s}(\mathbb{T})$ were restricted to the case $s>3 / 2$. Then, using functions spaces based on the linear group, Bourgain was able to prove global well-posedness in $L^{2}(\mathbb{T})$. Making 
use of the same spaces, and improving the nonlinear estimate, Kenig, Ponce and Vega (see [15]) proved local well-posedness in $H^{s}(\mathbb{T})$ for $s>-1 / 2$ (see Colliander et al. [7] for $s=-1 / 2$ ). After that, using a splitting into high and low Fourier frequencies of the solution, together with almost conserved quantities and rescaling arguments, Colliander et al. [7] were able to prove global existence in $H^{s}(\mathbb{T})$ for $s \geq-1 / 2$.

Using Bourgain's type spaces, we were able in [3] to prove local existence of solutions for (1.1) in the real line case, when the noise is a "localized space-time white noise", that is when its correlation function has the form

$$
\mathbb{E}\left(\phi \frac{\partial^{2} B}{\partial t \partial x}(t, x) \phi \frac{\partial^{2} B}{\partial t \partial x}(s, y)\right)=k(x) k(y) \delta_{x-y} \delta_{t-s}
$$

$k$ being an $L^{2}$ function. It is indeed hopeless in the real line case to be able to get even local existence of solutions in $H^{s}(\mathbb{R})$, with a pure space time white noise. The obstruction is not due to the lack of spatial regularity of the noise, but to its homogeneity (see [3]). In the periodic case, however, there is no such obstruction, and we are able to treat homogeneous noises, i.e. noises whose spatial correlation function depends only on $x-y$ (or such that $\phi$ is a convolution operator); also, thanks to the use of Bourgain's method adapted to Besov spaces, we are able to treat noises which have spatial correlations in $H^{s}$, $s>-1 / 2$. The main difficulty encountered in the application of Bourgain's method in our case, is that it needs time regularity of order $1 / 2$. However, it is well known that this regularity does not hold for Brownian motions unless Besov spaces are considered. This is why we use this method in the context of Besov spaces - see below for details. The problem of global existence of solutions for such noises in spaces with negative regularity is not considered here, but could probably be handled with the use of the method previously mentioned ([7]).

Before stating our result precisely, we introduce a few notations and assumptions.

We consider $\tilde{W}(t)=\frac{\partial B}{\partial x}$ a cylindrical Wiener process on $L^{2}(\mathbb{T})$ which may be written as $\tilde{W}(t)=\sum_{j \in \mathbb{N}} \beta_{j} e_{j}$ where $\left(e_{j}\right)_{j \in \mathbb{N}}$ is a complete orthonormal system in $L^{2}(\mathbb{T}),\left(\beta_{j}\right)_{j \in \mathbb{N}}$ is a sequence of mutually independent real valued Brownian motions in a fixed probability space $(\Omega, \mathcal{F}, \mathbb{P})$ associated with a filtration $\left(\mathcal{F}_{t}\right)_{t>0}$.

The process $W=\phi \tilde{W}$ is then a $\phi \phi^{*}$-Wiener process (recall that $\phi$ is a linear bounded operator in $\left.L^{2}(\mathbb{T})\right)$, i.e. $(W(t))_{t \geq 0}$ is a Gaussian process with law $\left(\mathcal{N}\left(0, t \phi \phi^{*}\right)_{t \geq 0}\right)$. 
We then consider equation (1.1) in its Itô form

$$
d u+\left(\partial_{x}^{3} u+u \partial_{x} u\right) d t=d W, x \in \mathbb{T}, t \geq 0,
$$

supplemented with the initial condition

$$
u(0, x)=u_{0}(x), x \in \mathbb{T} .
$$

Consider the Fourier transform

$$
\hat{f}(n)=\frac{1}{\sqrt{2 \pi}} \int_{\mathbb{T}} e^{i n x} f(x) d x
$$

for functions $f$ defined on $\mathbb{T}$, and let for $s \in \mathbb{R}, H^{s}(\mathbb{T})$ be the Sobolev space of functions $f$ such that the norm

$$
|f|_{H^{s}(\mathbb{T})}:=\left(\sum_{n \in \mathbb{Z}}\left(1+n^{2}\right)^{s}|\hat{f}(n)|^{2}\right)^{1 / 2}
$$

is finite. We also define, for $s \in \mathbb{R}$, the Besov space $B_{2,1}^{s}(\mathbb{T})$ as the space of functions $f$ defined on $\mathbb{T}$ for which the norm

$$
|f|_{B_{2,1}^{s}(\mathbb{T})}=|\hat{f}(0)|+\sum_{n \in \mathbb{N}} 2^{s n}\left(\sum_{2^{n-1} \leq\left|n^{\prime}\right| \leq 2^{n+1}}\left|\hat{f}\left(n^{\prime}\right)\right|^{2}\right)^{1 / 2}
$$

is finite.

Let $U(t)=e^{-t \partial_{x}^{3}}$ be the group associated with the linear equation on $L^{2}(\mathbb{T})$, that is $v(t)=U(t) u_{0}$ satisfies

$$
\left\{\begin{array}{l}
\partial_{t} v+\partial_{x}^{3} v=0 \\
v(0, x)=u_{0}(x), \quad x \in \mathbb{T} .
\end{array}\right.
$$

Then the solution of

$$
\left\{\begin{array}{l}
d w+\partial_{x}^{3} w d t=d W \\
w(0, x)=0, \quad x \in \mathbb{T}
\end{array}\right.
$$

is given by the stochastic convolution

$$
w(t)=\int_{0}^{t} U(t-s) d W(s)
$$

Note that $U(t)$ is a unitary group on $H^{s}(\mathbb{T})$ for any $s \in \mathbb{R}$, so that $w(t)$ lies in $H^{s}(\mathbb{T})$ almost surely if and only if $\phi \phi^{*}$ has finite trace from $L^{2}(\mathbb{T})$ into $H^{s}(\mathbb{T})$. This clearly holds in the case $\phi$ is the identical operator on $L^{2}(\mathbb{T})$ if and only if $s<-1 / 2$.

The difficulty in the use of Bourgain's spaces here is the smoothness in time. Indeed, let $Y^{s, b}$ be the space of functions $f$ such that $U(-t) f(t, \cdot) \in H^{s, b}$, where 
$H^{s, b}$ is a space-time Sobolev space, $s$ being the regularity in space, and $b$ the regularity in time (see [15] for a precise definition of $Y^{s, b}$ ). Then, as was proved in [15], the only possible value of $b$ for which a bilinear estimate holds, which allows to handle the nonlinear term $\partial_{x}\left(u^{2}\right)$ in the $\mathrm{KdV}$ equation using a straightforward iteration scheme, in the periodic case, is $b=1 / 2$. Writing then the expression of $w(t)$ defined by (1.4) as

$$
w(t)=\sum_{j \in \mathbb{N}} \int_{0}^{t} U(t-s)\left(\phi e_{j}\right) d \beta_{j}(s),
$$

one can compute the spatial Fourier transform of $h(t)=U(-t) w(t)$ :

$$
\hat{h}(t, n)=\sum_{j \in \mathbb{N}} \int_{0}^{t} e^{i s n^{3}} \widehat{\phi e}_{j}(n) d \beta_{j}(s) .
$$

But, there is no hope that this term lives in $H^{1 / 2}[0, T]$ in the time variable, because the Brownian motions $\beta_{j}$ do not. Indeed,

$$
\begin{aligned}
\mathbb{E}\left(|\hat{h}(t, n)|_{H_{t}^{1 / 2}}^{2}\right)= & \sum_{j \in \mathbb{N}}\left|\widehat{\phi e}_{j}(n)\right|^{2} \mathbb{E}\left|\int_{0}^{t} e^{i s n^{3}} d \beta_{j}(s)\right|_{H_{t}^{1 / 2}}^{2} \\
= & \sum_{j \in \mathbb{N}}\left|\widehat{\phi e}_{j}(n)\right|^{2}\left\{\mathbb{E} \int_{0}^{T}\left|\int_{0}^{t} e^{i s n^{3}} d \beta_{j}(s)\right|^{2} d t\right. \\
& \left.+\mathbb{E} \iint_{(0, T)^{2}} \frac{\left|\int_{0}^{t_{1}} e^{i s n^{3}} d \beta_{j}(s)-\int_{0}^{t_{2}} e^{i s n^{3}} d \beta_{j}(s)\right|^{2}}{\left|t_{1}-t_{2}\right|^{2}} d t_{1} d t_{2}\right\} .
\end{aligned}
$$

The first term in the right hand side above is obviously equal to $\frac{T^{2}}{2} \sum_{j \in \mathbb{N}}\left|\widehat{\phi e}_{j}(n)\right|^{2}$ while the contribution of each $j$ to the second term in the right hand side above is infinite, due to the fact that

$$
\mathbb{E}\left|\int_{t_{1}}^{t_{2}} e^{i s n^{3}} d \beta_{j}(s)\right|^{2}=\left|t_{2}-t_{1}\right| .
$$

However, $H^{1 / 2}$ is a limiting case concerning the regularity of the Brownian motion, as far as we are dealing with Sobolev spaces. It is then natural to try to replace Sobolev spaces here by other spaces which describe more precisely the regularity in time of the Brownian motions. This is exactly what we will do here, using Besov spaces instead of Sobolev spaces in time. Indeed, it is known (see [6], [17]) that the Brownian motion lies almost surely in $B_{p, q}^{1 / 2}([0, T])$ if and only if $1 \leq p<+\infty$ and $q=+\infty$. Trying to derive some bilinear estimate which would allow us to handle in the same time both $w(t)$ defined by (1.4) 
and the nonlinear term,

$$
\int_{0}^{t} U(t-s)\left(\partial_{x}\left(u^{2}\right)(s)\right) d s
$$

we were led to consider also Besov spaces in the space variable.

We now turn to give precise definitions of these spaces. We denote by $\langle.,$. the $L^{2}$ space-time duality product, that is

$$
\begin{aligned}
\langle f, g\rangle & =\int_{\mathbb{T}} \int_{\mathbb{R}} f(t, x) \overline{g(t, x)} d t d x \\
& =\sum_{n \in \mathbb{Z}} \int_{\mathbb{R}} \hat{f}(\tau, n) \overline{\hat{g}(\tau, n)} d \tau
\end{aligned}
$$

by the Plancherel formula; here, and in all what follows, we denote by $\hat{f}$ (resp. $\hat{g}$ ) the Fourier transform of $f$ (resp. $g$ ) with respect to both variables. We also use the notation $\langle\tau\rangle=\left(1+|\tau|^{2}\right)^{1 / 2}$, for $\tau \in \mathbb{R}$. The spaces that we will use are defined as follows. Consider first functions $f$ defined on $\mathbb{R} \times \mathbb{T}$ such that $f(., x) \in \mathcal{S}^{\prime}(\mathbb{R})$ for any $x \in \mathbb{T}$, and such that $\hat{f}(\tau, 0)=0$ for any $\tau \in \mathbb{R}$.

We denote by $X_{1,1}^{s, b}$ the space of such functions $f$ for which in addition the norm

$$
\begin{aligned}
|f|_{X_{1,1}^{s, b}}= & \sum_{n=0}^{\infty} 2^{s n} \sum_{k=0}^{\infty}\left(\sum_{2^{n-1} \leq\left|n^{\prime}\right| \leq 2^{n+1}} \int_{2^{k-1}}^{2^{k+1}}\left|\left\langle\tau-n^{\prime 3}\right\rangle^{b} \hat{f}\left(\tau, n^{\prime}\right)\right|^{2} d \tau\right)^{1 / 2} \\
& +\sum_{n=0}^{\infty} 2^{s n}\left(\sum_{2^{n-1} \leq\left|n^{\prime}\right| \leq 2^{n+1}} \int_{0}^{1}\left|\left\langle\tau-n^{\prime 3}\right\rangle^{b} \hat{f}\left(\tau, n^{\prime}\right)\right|^{2} d \tau\right)^{1 / 2}
\end{aligned}
$$

is finite. In the same way, we will denote by $X_{1, \infty}^{s, b}$ the space of such functions $f$ for which in addition the norm

$$
\begin{aligned}
|f|_{X_{1, \infty}^{s, b}}= & \sum_{n=0}^{\infty} 2^{s n} \sup _{k \in \mathbb{N}}\left(\sum_{2^{n-1} \leq\left|n^{\prime}\right| \leq 2^{n+1}} \int_{2^{k-1}}^{2^{k+1}}\left|\left\langle\tau-n^{\prime 3}\right\rangle^{b} \hat{f}\left(\tau, n^{\prime}\right)\right|^{2} d \tau\right)^{1 / 2} \\
& +\sum_{n=0}^{\infty} 2^{s n}\left(\sum_{2^{n-1} \leq\left|n^{\prime}\right| \leq 2^{n+1}} \int_{0}^{1}\left|\left\langle\tau-n^{\prime 3}\right\rangle^{b} \hat{f}\left(\tau, n^{\prime}\right)\right|^{2} d \tau\right)^{1 / 2}
\end{aligned}
$$

is finite.

The basic space in which we will solve the Cauchy problem for the stochastic $\mathrm{KdV}$ equation is $X_{1,1}^{s, b}$. However, we will make use, at intermediate steps, of other spaces of the same type : $\widetilde{X}_{1,1}^{s, b}\left(\operatorname{resp} . \widetilde{X}_{1, \infty}^{s, b}\right)$ is the space of functions $f$ such that $f(t, \cdot)=U(t) g(t, \cdot)$ with $g$ in the "space-time Besov space" $\left(B_{2,1}^{s, b}\right)_{x, t}$ 
(resp. $\left.\left(B_{2,1}^{s}\right)_{x}\left(B_{2, \infty}^{b}\right)_{t}\right)$, where $\left(B_{2,1}^{s, b}\right)_{x, t}$ is defined by the norm

$$
\begin{aligned}
|f|_{\left(B_{2,1}^{s, b}\right)_{x, t}=} & \sum_{n=0}^{\infty} \sum_{k=0}^{\infty} 2^{s n+k b}\left(\sum_{2^{n-1} \leq\left|n^{\prime}\right| \leq 2^{n+1}} \int_{2^{k-1}}^{2^{k+1}}\left|\hat{f}\left(\tau, n^{\prime}\right)\right|^{2} d \tau\right)^{1 / 2} \\
& +\sum_{n=0}^{\infty} 2^{s n}\left(\sum_{2^{n-1} \leq\left|n^{\prime}\right| \leq 2^{n+1}} \int_{0}^{1}\left|\hat{f}\left(\tau, n^{\prime}\right)\right|^{2} d \tau\right)^{1 / 2}
\end{aligned}
$$

and $\left(B_{2,1}^{s}\right)_{x}\left(B_{2, \infty}^{b}\right)_{t}$ is defined by the norm

$$
\begin{aligned}
|f|_{\left(B_{2,1}^{s}\right)_{x}\left(B_{2, \infty}^{b}\right) t}= & \sum_{n=0}^{\infty} \sup _{k \in \mathbb{N}} 2^{s n+k b}\left(\sum_{2^{n-1} \leq\left|n^{\prime}\right| \leq 2^{n+1}} \int_{2^{k-1}}^{2^{k+1}}\left|\hat{f}\left(\tau, n^{\prime}\right)\right|^{2} d \tau\right)^{1 / 2} \\
& +\sum_{n=0}^{\infty} 2^{s n}\left(\sum_{2^{n-1} \leq\left|n^{\prime}\right| \leq 2^{n+1}} \int_{0}^{1}\left|\hat{f}\left(\tau, n^{\prime}\right)\right|^{2} d \tau\right)^{1 / 2}
\end{aligned}
$$

Remark 1.1. Note that the spaces $X_{1,1}^{s, b}$ and $\widetilde{X}_{1,1}^{s, b}$ are different and there is no inclusion relation between them: an alternative definition of the norm in $\widetilde{X}_{1,1}^{s, b}$ is

$$
\begin{aligned}
|f|_{\widetilde{X}_{1,1}^{s, b}}= & \sum_{n=0}^{\infty} 2^{s n} \sum_{k=0}^{\infty}\left(\sum_{2^{n-1} \leq\left|n^{\prime}\right| \leq 2^{n+1}} \int_{2^{k-1} \leq\left|\tau-n^{\prime 3}\right| \leq 2^{k+1}}\left|\left\langle\tau-n^{\prime 3}\right\rangle^{b} \hat{f}\left(\tau, n^{\prime}\right)\right|^{2} d \tau\right)^{1 / 2} \\
& +\sum_{n=0}^{\infty} 2^{s n}\left(\sum_{2^{n-1} \leq\left|n^{\prime}\right| \leq 2^{n+1}} \int_{\left|\tau-n^{\prime 3}\right| \leq 1}\left|\left\langle\tau-n^{\prime 3}\right\rangle^{b} \hat{f}\left(\tau, n^{\prime}\right)\right|^{2} d \tau\right)^{1 / 2} ;
\end{aligned}
$$

here, the dyadic decomposition is made on $\left|\tau-n^{\prime 3}\right|$ and not on $|\tau|$. However, embeddings do hold between these spaces with some small loss of space regularity, as is stated in Lemma 1.1, at the end of this section.

Since all those definitions have to be used only locally in time, we will actually consider, for $T \geq 0$ fixed, the spaces $X_{1,1}^{s, b, T}$ and $X_{1, \infty}^{s, b, T}$ of restrictions on $[0, T]$ of functions of $X_{1,1}^{s, b}\left(\operatorname{resp} . X_{1, \infty}^{s, b}\right)$. They are endowed with the natural norm

$$
|f|_{X_{1,1}^{s, b, T}}=\inf \left\{|\tilde{f}|_{X_{1,1}^{s, b}}, \quad \tilde{f} \in X_{1,1}^{s, b} \text { and } f=\tilde{f}_{\left.\right|_{[0, T]}}\right\}
$$

and the equivalent for $X_{1, \infty}^{s, b, T}$.

To handle the integral estimate in Duhamel's formula, we will need to make use, as is classical, of another space which is defined as the space of zero 
(spatial) mean functions with finite corresponding norm, where

$$
|f|_{Y_{s}}=\sum_{n=0}^{\infty} 2^{s n}\left(\sum_{2^{n-1} \leq\left|n^{\prime}\right| \leq 2^{n+1}}\left(\int_{\mathbb{R}} \frac{\left|\hat{f}\left(\tau, n^{\prime}\right)\right|}{\left\langle\tau-n^{\prime 3}\right\rangle} d \tau\right)^{2}\right)^{1 / 2}
$$

A local space $Y_{s, T}$ is also defined, in the same way as for $X_{1,1}^{s, 1 / 2, T}$.

In all the paper, we will use the notation $\left|n^{\prime}\right| \sim 2^{n}$ for $2^{n-1} \leq\left|n^{\prime}\right| \leq 2^{n+1}$, and $|\tau| \sim 2^{k}$ for $2^{k-1} \leq|\tau| \leq 2^{k+1}$ if $k \geq 1$ and $|\tau| \leq 2$ if $k=0$.

As previously mentioned, we will be led to assume ${ }^{1}$ that the operator $\phi$ is a Hilbert-Schmidt operator (or equivalently that $\phi \phi^{*}$ has finite trace) from $L^{2}(\mathbb{T})$ into $H^{s}(\mathbb{T})$ for some negative $s$ with $s>-1 / 2$. We will denote by $L_{2}^{0, s}$ the space of such operators, which is endowed by its natural norm :

$$
\|\phi\|_{L_{2}^{0, s}}=\left(\sum_{i \in \mathbb{N}}\left|\phi e_{i}\right|_{H^{s}}^{2}\right)^{1 / 2}
$$

where $\left(e_{i}\right)_{i \in \mathbb{N}}$ is any complete orthonormal system in $L^{2}(\mathbb{T})$. For convenience, in all what follows, we take as $\left(e_{i}\right)_{i \in \mathbb{N}}$ the usual complete orthonormal system of $L^{2}(\mathbb{T})$ given by

$$
\begin{aligned}
& e_{2 k}(x)=\frac{1}{\sqrt{\pi}} \cos k x, \quad k \geq 1, \quad e_{0}(x)=\frac{1}{\sqrt{2 \pi}} \\
& e_{2 k+1}(x)=\frac{1}{\sqrt{\pi}} \sin k x .
\end{aligned}
$$

We consider the mild form of equations (1.2), (1.3), that is

$$
u(t)=U(t) u_{0}-\frac{1}{2} \int_{0}^{t} U(t-s) \partial_{x}\left(u^{2}(s)\right) d s+\int_{0}^{t} U(t-s) d W(s) .
$$

Our main result, which concerns local existence in a situation where $W$ is arbitrarily close to a cylindrical Wiener process, is the following.

Theorem 1.1. Assume that $\operatorname{Im} \phi \subset \operatorname{span}\left\{e_{j}, j \geq 1\right\}$ and that $\phi \in L_{2}^{0, s}$ for some $s$ with $s>-1 / 2$. Let $u_{0}$ be $\mathcal{F}_{0}$-measurable, with $u_{0}$ in the Besov space $B_{2,1}^{\sigma}(\mathbb{T})$ a.s., for some $\sigma$ with $-1 / 2 \leq \sigma<s$; then there is a stopping time $T_{\omega}>0$ and a unique process $u$ solution of the forced KdV equation (1.5) which satisfies

$$
u \in C\left(\left[0, T_{\omega}\right] ; B_{2,1}^{\sigma}(\mathbb{T})\right) \cap X_{1,1}^{\sigma, 1 / 2, T_{\omega}} \text { a.s. }
$$

Remark 1.2. The assumption $\operatorname{Im} \phi \subset \operatorname{span}\left\{e_{j}, j \geq 1\right\}$ says that the spatial mean of the noise is zero at any time. This assumption is necessary to perform

\footnotetext{
${ }^{1}$ note that this assumption excludes the identical operator on $L^{2}(\mathbb{T})$
} 
the fixed point procedure, because we work in a space of functions with zero spatial mean. We will actually remove this assumption at the end of the paper (see Proposition 4.2) by changing the unknown function $u$ and the noise. At that place, we will have to deal with a non Gaussian noise.

Remark 1.3. One can show by using classical arguments, and looking more carefully into the proof of Proposition 3.1 (see Section 3) that the regularity is preserved in Theorem 1.1, i.e. if $\phi \in L_{2}^{0, s}$ and $u_{0} \in B_{2,1}^{\sigma}(\mathbb{T})$ with $-1 / 2 \leq$ $\sigma^{\prime} \leq \sigma<s$, then the existence times of the solution in $B_{2,1}^{\sigma^{\prime}}(\mathbb{T})$ and in $B_{2,1}^{\sigma}(\mathbb{T})$ are the same.

Naturally, when the noise is such that the Wiener process lies in $L^{2}(\mathbb{T})$, we get a global existence result thanks to the invariance of the $L^{2}$ norm for the deterministic equation and the embedding $L^{2}(\mathbb{T}) \subset B_{2,1}^{\sigma}(\mathbb{T})$, for any $\sigma<0$.

Theorem 1.2. Assume that in addition, $\phi \in L_{2}^{0,0} ;$ then if $u_{0} \in L^{2}\left(\Omega ; L^{2}(\mathbb{T})\right)$, the solution given by Theorem 1.1 is globally defined in time and lies in $L^{2}\left(\Omega ; L^{\infty}\left(0, T ; L^{2}(\mathbb{T})\right)\right)$ and in $C\left(\mathbb{R}^{+} ; B_{2,1}^{\sigma}(\mathbb{T})\right)$ a.s. for any $T>0$ and $\sigma<0$.

As was previously mentioned, Theorem 1.1 allows to handle a situation arbitrarily close to the space time white noise case, since this latter case corresponds to $\phi=\mathrm{id}$, which is a Hilbert-Schmidt operator from $L^{2}(\mathbb{T})$ into $H^{s}(\mathbb{T})$ for any $s<-1 / 2$. Theorem 1.1 will be proved by using a fixed point argument in the space $X_{1,1}^{\sigma, 1 / 2, T}$ for $T$ small enough. We need the assumption $s>-1 / 2$ because we will need that $s>\sigma \geq-1 / 2$. Indeed, to show that the fixed point works, we will first prove that the stochastic integral lies almost surely in $X_{1, \infty}^{\sigma, 1 / 2}$. At that point, we have already lost some spatial regularity. We then prove a bilinear estimate allowing us to handle such a term as $\partial_{x}(f g)$ with $f \in X_{1,1}^{\sigma, 1 / 2}$ and $g \in X_{1, \infty}^{\sigma, 1 / 2}$. To treat the term $\partial_{x}\left(g^{2}\right)$ in the same space, we again have to sacrifice an arbitrarily small amount of spatial regularity.

It is not difficult to see that when $\phi=\mathrm{id}$, the stochastic integral $w(t)$ given by (1.4) lies almost surely in $X_{\infty, \infty}^{-1 / 2,1 / 2}$, where this latest space is defined by changing the norm in the definition of $X_{1, \infty}^{-1 / 2,1 / 2}$ in an obvious way. Unfortunately, a bilinear estimate which would handle terms like $\partial_{x}\left(g^{2}\right)$ in $X_{\infty, \infty}^{-1 / 2,-1 / 2}$ with $g$ in $X_{\infty, \infty}^{-1 / 2,1 / 2}$ seems to fail.

The paper is organized as follows. In Section 2, we prove an estimate which shows that the stochastic integral lives in $X_{1, \infty}^{\sigma, 1 / 2}$ almost surely when $\phi$ is in $L_{2}^{0, s}$ with $\sigma<s$ (we will actually prove that the stochastic integral lies in 
$\widetilde{X}_{1, \infty}^{\sigma, 1 / 2}$, which is enough thanks to Lemma 1.1 below). This result is based on the works of Cieselskii and Roynette ([6], [17]), but we will use a different characterization of Besov spaces than in [17].

In Section 3, we prove some bilinear estimates which are needed in the proof of Theorem 1.1. The main one is an estimate of $\partial_{x}(f g)$ in $X_{1,1}^{\sigma,-1 / 2}$ when $f \in X_{1,1}^{\sigma, 1 / 2}$ and $g \in X_{1, \infty}^{\sigma, 1 / 2}$. Other easier bilinear estimates are proved in that Section too.

Section 4 is devoted to the proof of Theorems 1.1 and 1.2. Once we have the bilinear estimates in hand, together with the estimate on the stochastic integral, it mainly remains to prove that we gain one degree of regularity in time when passing from $\partial_{x}(f g)$ to $\int_{0}^{t} U(t-s) \partial_{x}(f g)(s) d s$. The proof of this fact has to be done because we do not stand in the usual context of Sobolev spaces, but we deal with Besov spaces. However, the proof closely follows that of the Sobolev case.

We end the present section by giving the lemma relating the spaces $X_{1,1}^{s, b}$ and $\widetilde{X}_{1, \infty}^{s, b}$.

Lemma 1.1. For any $s_{1}>s_{2}>s_{3}$,

$$
\widetilde{X}_{1,1}^{s_{1}, b} \subset X_{1,1}^{s_{2}, b} \subset \widetilde{X}_{1,1}^{s_{3}, b} \quad \text { and } \quad \widetilde{X}_{1, \infty}^{s_{1}, b} \subset X_{1, \infty}^{s_{2}, b} \subset \widetilde{X}_{1, \infty}^{s_{3}, b}
$$

Proof. We only show that $\widetilde{X}_{1, \infty}^{s_{1}, b} \subset X_{1, \infty}^{s_{2}, b}$, all the other embeddings are proved similarly. Let $f \in \widetilde{X}_{1, \infty}^{s_{1}, b}$ and let us decompose the norm of $f$ in $X_{1, \infty}^{s_{2}, b}$ as

$$
\begin{aligned}
|f|_{X_{1, \infty}^{s_{2}, b}} & \leq \sum_{n \in \mathbb{N}} 2^{s_{2} n} \sup _{k<3 n-4}\left(\sum_{\left|n^{\prime}\right| \sim 2^{n}} \int_{|\tau| \sim 2^{k}}\left\langle\tau-n^{\prime 3}\right\rangle^{2 b}\left|\hat{f}\left(\tau, n^{\prime}\right)\right|^{2} d \tau\right)^{1 / 2} \\
& +\sum_{n \in \mathbb{N}} 2^{s_{2} n} \sup _{3 n-4 \leq k \leq 3 n+4}\left(\sum_{\left|n^{\prime}\right| \sim 2^{n}} \int_{|\tau| \sim 2^{k}}\left\langle\tau-n^{\prime 3}\right\rangle^{2 b}\left|\hat{f}\left(\tau, n^{\prime}\right)\right|^{2} d \tau\right)^{1 / 2} \\
& +\sum_{n \in \mathbb{N}} 2^{s_{2} n} \sup _{k>3 n+4}\left(\sum_{\left|n^{\prime}\right| \sim 2^{n}} \int_{|\tau| \sim 2^{k}}\left\langle\tau-n^{\prime 3}\right\rangle^{2 b}\left|\hat{f}\left(\tau, n^{\prime}\right)\right|^{2} d \tau\right)^{1 / 2} \\
& \leq I+I I+I I I .
\end{aligned}
$$


If $k>3 n+4,\left|n^{\prime}\right| \sim 2^{n}$ and $|\tau| \sim 2^{k}$, then $\frac{1}{8}|\tau| \leq\left|\tau-n^{\prime 3}\right| \leq \frac{3}{2}|\tau|$, hence we easily have

$$
\begin{aligned}
I I I & \leq C \sum_{n \in \mathbb{N}} 2^{s_{2} n} \sup _{k \in \mathbb{N}}\left(\sum_{\left|n^{\prime}\right| \sim 2^{n}} \int_{\left|\tau-n^{\prime 3}\right| \sim 2^{k}}\left\langle\tau-n^{\prime 3}\right\rangle^{2 b}\left|\hat{f}\left(\tau, n^{\prime}\right)\right|^{2} d \tau\right)^{1 / 2} \\
& \leq C|f|_{\widetilde{X}_{1, \infty}^{s_{2}, b}}
\end{aligned}
$$

On the other hand, if $k<3 n-4,\left|n^{\prime}\right| \sim 2^{n}$ and $|\tau| \sim 2^{k}$, then $2^{3 n-4} \leq$ $\left|\tau-n^{\prime 3}\right| \leq 2^{3 n+4}$; hence

$$
\begin{aligned}
I & \leq \sum_{n \in \mathbb{N}} 2^{s_{2} n}\left(\sum_{\left|n^{\prime}\right| \sim 2^{n}} \int_{2^{3 n-4} \leq\left|\tau-n^{\prime 3}\right| \leq 2^{3 n+4}}\left\langle\tau-n^{\prime 3}\right\rangle^{2 b}\left|\hat{f}\left(\tau, n^{\prime}\right)\right|^{2} d \tau\right)^{1 / 2} \\
& \leq 8|f|_{\widetilde{X}_{1, \infty}^{s_{2}, b}}
\end{aligned}
$$

Finally, if $3 n-4 \leq k \leq 3 n+4,\left|n^{\prime}\right| \sim 2^{n}$ and $|\tau| \sim 2^{k}$, then $0 \leq\left|\tau-n^{\prime 3}\right| \leq 2^{3 n+6}$, hence

$$
\begin{aligned}
I I & \leq \sum_{n \in \mathbb{N}} 2^{s_{2} n}\left(\sum_{\left|n^{\prime}\right| \sim 2^{n}} \sum_{k=0}^{3 n+5} \int_{\left|\tau-n^{\prime 3}\right| \sim 2^{k}}\left\langle\tau-n^{\prime 3}\right\rangle^{2 b}\left|\hat{f}\left(\tau, n^{\prime}\right)\right|^{2} d \tau\right)^{1 / 2} \\
& \leq \sum_{n \in \mathbb{N}} 2^{s_{2} n}(3 n+5) \sup _{k \in \mathbb{N}}\left(\sum_{\left|n^{\prime}\right| \sim 2^{n}} \int_{\left|\tau-n^{\prime 3}\right| \sim 2^{k}}\left\langle\tau-n^{\prime 3}\right\rangle^{2 b}\left|\hat{f}\left(\tau, n^{\prime}\right)\right|^{2} d \tau\right)^{1 / 2} \\
& \leq C \sum_{n \in \mathbb{N}} 2^{s_{1} n} \sup _{k \in \mathbb{N}}\left(\sum_{\left|n^{\prime}\right| \sim 2^{n}} \int_{\left|\tau-n^{\prime 3}\right| \sim 2^{k}}\left\langle\tau-n^{\prime 3}\right\rangle^{2 b}\left|\hat{f}\left(\tau, n^{\prime}\right)\right|^{2} d \tau\right)^{1 / 2}
\end{aligned}
$$

since $s_{2}<s_{1}$. The result follows.

\section{Estimate on the STOCHASTIC INTEGRAL}

In this section, we prove an estimate on the stochastic integral - that is the last term in (1.5) - which will enable us to use a fixed point procedure to solve (1.5) in an appropriate space of functions of the space and time variables. This latest space will actually be of the form $X_{1,1}^{\sigma, 1 / 2}$ for some well chosen $\sigma$.

Although, for the sake of clarity, we did not assume that the covariance operator $\phi \phi^{*}$ of the noise could be random or could depend on the time variable $t$ in Theorem 1.1, we will state here a proposition where $\phi$ is allowed to depend both on $t$ and $\omega$, but under the condition that the $L_{2}^{0, \sigma^{\prime}}$ norm of $\phi(\cdot)$ is bounded in both $t$ and $\omega$. This will indeed be useful in order to prove that our result generalizes to the case where the noise does not have a zero spatial mean value (see Proposition 4.2). 
We need to use a cut-off function in the time variable: we consider a function $\theta: \mathbb{R} \longrightarrow \mathbb{R}^{+}$such that $\theta(t) \equiv 0$ for $t \leq-1$, and $t \geq 2, \theta(t) \equiv 1$ for $t \in[0,1]$, and $\theta \in C_{0}^{\infty}(\mathbb{R})$.

Also, to state precisely our estimate on the stochastic integral, we define for $n \in \mathbb{N}$, the operator $\Delta_{n}$ acting on $L^{2}(\mathbb{T})$ by

$$
\widehat{\Delta_{n} u}\left(n^{\prime}\right)=\mathbb{1}_{\left\{2^{n-1} \leq\left|n^{\prime}\right| \leq 2^{n+1}\right\}} \hat{u}\left(n^{\prime}\right),
$$

for $u \in L^{2}(\mathbb{T})$ and for any $n^{\prime} \in \mathbb{Z}$.

We now state our proposition.

Proposition 2.1. Let $s^{\prime} \in \mathbb{R}$, and assume that $\phi$ is predictable and lies in $L^{\infty}\left([0, T] \times \Omega ; L_{2}^{0, s^{\prime}}\right)$ for some $T$ with $0<T \leq 1$; let $\theta$ and $\Delta_{n}$ be as above; then the stochastic integral $w(t)$ defined by (1.4) satisfies for any $\sigma^{\prime}<\sigma<s^{\prime}$ : $\theta w \in L^{1}\left(\Omega ; X_{1, \infty}^{\sigma^{\prime}, 1 / 2, T}\right)$ and

$$
\begin{aligned}
\mathbb{E}\left(|\theta w|_{X_{1, \infty}^{\sigma^{\prime}, 1 / 2, T}}\right) & \leq C(\theta) \sum_{n \in \mathbb{N}}\left\|\Delta_{n} \phi\right\|_{L^{\infty}\left([0, T] \times \Omega ; L_{2}^{0, \sigma}\right)} \\
& \leq C\left(\theta, \sigma, s^{\prime}\right)\|\phi\|_{L^{\infty}\left([0, T] \times \Omega ; L_{2}^{0, s^{\prime}}\right)}
\end{aligned}
$$

where $C(\theta)$ is a constant depending only on the function $\theta$.

Proof. We first prove that $\theta w \in L^{1}\left(\Omega ; \widetilde{X}_{1, \infty}^{\sigma, 1 / 2, T}\right)$ and that

$$
\mathbb{E}\left(|\theta w|_{\widetilde{X}_{1, \infty}^{\sigma, 1 / 2, T}}\right) \leq C(\theta) \sum_{n \in \mathbb{N}}\left\|\Delta_{n} \phi\right\|_{L^{\infty}\left([0, T] \times \Omega ; L_{2}^{0, \sigma}\right)}
$$

and then make use of Lemma 1.1.

Let $g(t, \cdot)=\theta(t) \int_{0}^{t} U(-s) d W(s)$ so that $\theta(t) w(t)=U(t) g(t, \cdot)$; we also set for $s \in \mathbb{R}, n \in \mathbb{Z}$ and $\ell \in \mathbb{N}$ :

$$
\varphi_{n, \ell}(s)=\left\{\begin{array}{l}
0 \text { if } s<0 \text { or } s \geq T \\
\widehat{\phi(s) e_{\ell}}(n) \text { if } s \in[0, T]
\end{array}\right.
$$

and we assume that each $\beta_{\ell}$ has been extended to a Brownian motion on $\mathbb{R}$, in such a way that the family $\left(\beta_{\ell}\right)_{\ell \in \mathbb{N}}$ is still an independent family. We then have, for any $t \in[0, T]$ and $n \in \mathbb{Z}$ :

$$
\mathcal{F}_{n} g(t)(n)=\sum_{\ell \in \mathbb{N}} \theta(t) I_{n, \ell}(t)
$$

with $I_{n, \ell}(t)=\int_{-\infty}^{t} \theta(s) e^{i n s^{3}} \varphi_{n, \ell}(s) d \beta_{\ell}(s), \mathcal{F}_{n}$ being the Fourier transform in space. 
In view of the equivalent definition of the space $\widetilde{X}_{1, \infty}^{\sigma, 1 / 2}$, we have to show that

$$
\begin{aligned}
& \mathbb{E}\left(\sum_{n=0}^{\infty} \sup _{k \in \mathbb{N}} 2^{\sigma n+k / 2}\left(\sum_{\left|n^{\prime}\right| \sim 2^{n}} \int_{|\tau| \sim 2^{k}}\left|\hat{g}\left(\tau, n^{\prime}\right)\right|^{2} d \tau\right)^{1 / 2}\right) \\
& +\mathbb{E}\left(\sum_{n=0}^{\infty} 2^{\sigma n}\left(\sum_{\left|n^{\prime}\right| \sim 2^{n}} \int_{|\tau| \leq 1}\left|\hat{g}\left(\tau, n^{\prime}\right)\right|^{2} d \tau\right)^{1 / 2}\right) \\
& \leq C(\theta) \sum_{n=0}^{\infty}\left\|\Delta_{n} \phi\right\|_{L^{\infty}\left([0, T] \times \Omega ; L_{2}^{0, \sigma}\right)} .
\end{aligned}
$$

We first estimate the second term in (2.1).

$$
\begin{aligned}
& \mathbb{E}\left(\sum_{n=0}^{\infty} 2^{\sigma n}\left(\sum_{\left|n^{\prime}\right| \sim 2^{n}} \int_{|\tau| \leq 1}\left|\sum_{\ell \in \mathbb{N}} \widehat{\theta I_{n^{\prime}, \ell}}(\tau)\right|^{2} d \tau\right)^{1 / 2}\right) \\
& \leq \sum_{n=0}^{\infty} 2^{\sigma n}\left(\sum_{\left|n^{\prime}\right| \sim 2^{n}} \mathbb{E} \int_{|\tau| \leq 1}\left|\sum_{\ell \in \mathbb{N}} \int_{\mathbb{R}} \theta(t) \int_{-\infty}^{t} \theta(s) e^{i s n^{\prime 3}} \varphi_{n^{\prime}, \ell}(s) d \beta_{\ell}(s) e^{-i \tau t} d t\right|^{2} d \tau\right)^{1 / 2} \\
& \leq \sum_{n=0}^{\infty} 2^{\sigma n}\left(\sum_{\left|n^{\prime}\right| \sim 2^{n}} \int_{|\tau| \leq 1} \mathbb{E}\left|\sum_{\ell \in \mathbb{N}} \int_{\mathbb{R}} \theta(s) \varphi_{n^{\prime}, \ell}(s) e^{i s n^{\prime 3}} \int_{s}^{+\infty} \theta(t) e^{-i \tau t} d t d \beta_{\ell}(s)\right|^{2} d \tau\right)^{1 / 2}
\end{aligned}
$$

and using the independence of the $\left(\beta_{\ell}\right)_{\ell \in \mathbb{N}}$, the above term is bounded by

$$
\begin{aligned}
& \sum_{n=0}^{\infty} 2^{\sigma n}\left(\sum_{\left|n^{\prime}\right| \sim 2^{n}} \int_{|\tau| \leq 1} \sum_{\ell \in \mathbb{N}} \int_{\mathbb{R}} \mathbb{E}\left(\theta^{2}(s)\left|\varphi_{n^{\prime}, \ell}(s)\right|^{2}\right)\left|\int_{s}^{+\infty} \theta(t) e^{-i \tau t} d t\right|^{2} d s d \tau\right)^{1 / 2} \\
& \leq 2|\theta|_{L^{1}(\mathbb{R})}^{2}|\theta|_{L^{2}(\mathbb{R})}^{2} \sum_{n=0}^{+\infty} 2^{\sigma n} \sup _{s \in \mathbb{R}}\left|\mathbb{E}\left(\sum_{\ell \in \mathbb{N}} \sum_{\left|n^{\prime}\right| \sim 2^{n}}\left|\varphi_{n^{\prime}, \ell}(s)\right|^{2}\right)\right|^{1 / 2} \\
& \leq C(\theta) \sum_{n=0}^{+\infty} \sup _{s \in \mathbb{R}}\left|\mathbb{E}\left(\left\|\Delta_{n} \phi(s)\right\|_{L_{2}^{0, \sigma}}^{2}\right)\right|^{1 / 2} \\
& \leq C(\theta) \sum_{n=0}^{+\infty}\left\|\Delta_{n} \phi(\cdot)\right\|_{L^{\infty}\left([0, T] \times \Omega ; L_{2}^{0, \sigma}\right)}
\end{aligned}
$$

and this proves the estimate on the second term in (2.1).

In what follows, we assume that $|\tau| \geq 1 / 2$; by the stochastic Fubini Theorem and using an integration by parts, we easily get for $n \in \mathbb{Z}, \ell \in \mathbb{N}$ and $|\tau| \geq 1 / 2$ :

$$
\widehat{\theta I_{n, \ell}}(\tau)=A_{n, \ell}(\tau)+B_{n, \ell}(\tau)
$$

with

$$
A_{n, \ell}(\tau)=\int_{\mathbb{R}} \theta^{2}(s) e^{i s n^{3}} \varphi_{n, \ell}(s) \frac{e^{-i \tau s}}{i \tau} d \beta_{\ell}(s)
$$


and

$$
B_{n, \ell}(\tau)=\int_{\mathbb{R}} \theta(s) e^{i s n^{3}} \varphi_{n, \ell}(s) \int_{s}^{+\infty} \theta^{\prime}(t) \frac{e^{-i \tau t}}{i \tau} d t d \beta_{\ell}(s) .
$$

Hence, two terms will be involved in the estimate of the second term in (2.1), which are

$$
I=\mathbb{E}\left(\sum_{n=0}^{\infty} \sup _{k \in \mathbb{N}} 2^{\sigma n+k / 2}\left(\sum_{\left|n^{\prime}\right| \sim 2^{n}} \int_{|\tau| \sim 2^{k}}\left|\sum_{\ell=0}^{\infty} A_{n^{\prime}, \ell}(\tau)\right|^{2} d \tau\right)^{1 / 2}\right)
$$

and

$$
I I=\mathbb{E}\left(\sum_{n=0}^{\infty} \sup _{k \in \mathbb{N}} 2^{\sigma n+k / 2}\left(\sum_{\left|n^{\prime}\right| \sim 2^{n}} \int_{|\tau| \sim 2^{k}}\left|\sum_{\ell=0}^{\infty} B_{n^{\prime}, \ell}(\tau)\right|^{2} d \tau\right)^{1 / 2}\right) .
$$

We may assume that $\sigma=0$, replacing in the estimate we want to prove $\varphi_{n^{\prime}, \ell}$ by $2^{\sigma n} \varphi_{n^{\prime}, \ell}$. We first estimate the second term above. With this aim in view, we first write for $k \geq 0$ and $n \geq 0$ :

$$
\begin{aligned}
& \mathbb{E}\left(2^{k} \sum_{\left|n^{\prime}\right| \sim 2^{n}} \int_{|\tau| \sim 2^{k}}\left|\sum_{\ell=0}^{\infty} B_{n^{\prime}, \ell}(\tau)\right|^{2} d \tau\right) \\
& =2^{k} \int_{|\tau| \sim 2^{k}} \sum_{\left|n^{\prime}\right| \sim 2^{n}} \mathbb{E}\left|\sum_{\ell=0}^{\infty} \int_{\mathbb{R}} \theta(s) e^{i s n^{\prime 3}} \varphi_{n^{\prime}, \ell}(s) \int_{s}^{+\infty} \theta^{\prime}(t) \frac{e^{-i \tau t}}{i \tau} d t d \beta_{\ell}(s)\right|^{2} d \tau \\
& =2^{k} \int_{|\tau| \sim 2^{k}} \sum_{\ell=0}^{\infty} \sum_{\left|n^{\prime}\right| \sim 2^{n}} \int_{\mathbb{R}}|\theta(s)|^{2} \mathbb{E}\left(\left|\varphi_{n^{\prime}, \ell}(s)\right|^{2}\right)\left|\int_{s}^{+\infty} \theta^{\prime}(t) \frac{e^{-i \tau t}}{i \tau} d t\right|^{2} d s d \tau
\end{aligned}
$$

where we have used again the independence of the family $\left(\beta_{\ell}\right)_{\ell \geq 0}$. Now, for $|\tau|$ in $\left[2^{k-1}, 2^{k+1}\right]$, we have

$$
\left|\int_{s}^{+\infty} \theta^{\prime}(t) \frac{e^{-i \tau t}}{i \tau} d t\right| \leq\left|\frac{\theta^{\prime}(s)}{\tau^{2}}\right|+\left|\int_{s}^{+\infty} \theta^{\prime \prime}(t) \frac{e^{-i \tau t}}{\tau^{2}} d t\right| \leq \frac{C(\theta)}{2^{2 k}}
$$

hence we get for $k, n \geq 0$ :

$$
\begin{aligned}
& \mathbb{E}\left(2^{k} \sum_{\left|n^{\prime}\right| \sim 2^{n}} \int_{|\tau| \sim 2^{k}}\left|\sum_{\ell=0}^{\infty} B_{n^{\prime}, \ell}(\tau)\right|^{2} d \tau\right) \\
& \leq C(\theta) 2^{-3 k} \int_{|\tau| \sim 2^{k}}\left|\mathbb{E}\left(\left\|\Delta_{n} \phi(s)\right\|_{L_{2}^{0,0}}^{2}\right)\right|_{L_{s}^{\infty}} d \tau \\
& \leq C(\theta) 2^{-2 k}\left\|\Delta_{n} \phi(\cdot)\right\|_{L^{\infty}\left([0, T] \times \Omega ; L_{2}^{0,0}\right)}^{2},
\end{aligned}
$$


and using Cauchy-Schwarz inequality, we get

$$
\begin{aligned}
& I I=\sum_{n=0}^{+\infty} \sup _{k \in \mathbb{N}} \mathbb{E}\left(2^{k / 2}\left(\sum_{\left|n^{\prime}\right| \sim 2^{n}} \int_{|\tau| \sim 2^{k}}\left|\sum_{\ell=0}^{\infty} B_{n^{\prime}, \ell}(\tau)\right|^{2} d \tau\right)^{1 / 2}\right) \\
& \leq \sum_{n=0}^{\infty} \sum_{k \in \mathbb{N}}\left(\mathbb{E}\left(2^{k} \sum_{\left|n^{\prime}\right| \sim 2^{n}} \int_{|\tau| \sim 2^{k}}\left|\sum_{\ell=0}^{\infty} B_{n^{\prime}, \ell}(\tau)\right|^{2} d \tau\right)\right)^{1 / 2} \\
& \leq C(\theta) \sum_{n=0}^{\infty} \sum_{k \in \mathbb{N}} 2^{-k}\left\|\Delta_{n} \phi(\cdot)\right\|_{L^{\infty}\left([0, T] \times \Omega ; L_{2}^{0,0}\right)} \\
& \leq C(\theta) \sum_{n=0}^{\infty}\left\|\Delta_{n} \phi(\cdot)\right\|_{L^{\infty}\left([0, T] \times \Omega ; L_{2}^{0,0}\right)} .
\end{aligned}
$$

Our aim is now to estimate $I$. We set

$$
A_{n^{\prime}, \ell}(\tau, s)=\int_{-\infty}^{s} \theta^{2}(t) e^{i t n^{\prime 3}} \varphi_{n^{\prime}, \ell}(t) \frac{e^{-i \tau t}}{i \tau} d \beta_{\ell}(t)
$$

so that

$$
A_{n^{\prime}, \ell}(\tau)=\int_{-\infty}^{+\infty} d A_{n^{\prime}, \ell}(\tau, s)
$$

Moreover, using the Itô formula, we have

$$
\begin{aligned}
& \left|\sum_{\ell=0}^{+\infty} A_{n^{\prime}, \ell}(\tau)\right|^{2}=\int_{\mathbb{R}} d\left|\sum_{\ell \in \mathbb{N}} A_{n^{\prime}, \ell}(\tau, t)\right|^{2} \\
& =2 \operatorname{Re}\left(\sum_{\ell, m=0}^{\infty} \int_{\mathbb{R}} \int_{-\infty}^{t} \theta^{2}(s) e^{i s n^{\prime 3}} \varphi_{n^{\prime}, \ell}(s) \frac{e^{-i \tau s}}{i \tau} d \beta_{\ell}(s)\right. \\
& \left.\quad \times \theta^{2}(t) e^{-i t n^{\prime 3}} \varphi_{n^{\prime}, m}(t) \frac{e^{i \tau t}}{-i \tau} d \beta_{m}(t)\right)+\sum_{\ell \in \mathbb{N}} \int_{\mathbb{R}} \theta^{4}(t) \frac{\varphi_{n^{\prime}, \ell}^{2}(t)}{\tau^{2}} d t \\
& =I_{n^{\prime}}^{1}(\tau)+I_{n^{\prime}}^{2}(\tau) .
\end{aligned}
$$


Hence, again, two terms are involved in the estimate of $I$. The estimate of the second term is immediate. Indeed, we have

$$
\begin{aligned}
& \mathbb{E}\left(\sum_{n=0}^{\infty} \sup _{k \in \mathbb{N}} 2^{k / 2}\left(\sum_{\left|n^{\prime}\right| \sim 2^{n}} \int_{|\tau| \sim 2^{k}} I_{n^{\prime}}^{2}(\tau) d \tau\right)^{1 / 2}\right) \\
& =\mathbb{E}\left(\sum_{n=0}^{\infty} \sup _{k \in \mathbb{N}} 2^{k / 2}\left(\sum_{\left|n^{\prime}\right| \sim 2^{n}} \int_{|\tau| \sim 2^{k}} \sum_{\ell \in \mathbb{N}} \int_{\mathbb{R}} \theta^{4}(t) \frac{\varphi_{n^{\prime}, \ell}^{2}(\tau)}{\tau^{2}} d t d \tau\right)^{1 / 2}\right) \\
& \leq C(\theta) \sum_{n=0}^{\infty} \sup _{k \in \mathbb{N}} 2^{k / 2}\left(\int_{|\tau| \sim 2^{k}} \frac{1}{\tau^{2}}\left|\sum_{\left|n^{\prime}\right| \sim 2^{n}} \sum_{\ell \in \mathbb{N}} \varphi_{n^{\prime}, \ell}^{2}(\cdot)\right|_{L^{\infty}([0, T] \times \Omega)} d \tau\right)^{1 / 2} \\
& \leq C(\theta) \sum_{n=0}^{\infty}\left\|\Delta_{n} \phi(\cdot)\right\|_{L^{\infty}\left([0, T] \times \Omega ; L_{2}^{0,0}\right)}
\end{aligned}
$$

In order to estimate the contribution of the stochastic integral, i.e. of $I_{n^{\prime}}^{1}(\tau)$ in the bound of $I$, we start with the following estimate.

$$
\begin{aligned}
& \mathbb{E}\left(\left|\sum_{\left|n^{\prime}\right| \sim 2^{n}} \int_{|\tau| \sim 2^{k}} I_{n^{\prime}}^{1}(\tau) d \tau\right|^{2}\right) \\
& =\mathbb{E}\left(\mid \sum_{\left|n^{\prime}\right| \sim 2^{n}} \int_{|\tau| \sim 2^{k}} 2 \operatorname{Re}\left(\sum_{\ell, m=0}^{\infty} \int_{\mathbb{R}} \int_{-\infty}^{t} \theta^{2}(s) e^{i s n^{\prime 3}} \varphi_{n^{\prime}, \ell}(s) \frac{e^{-i \tau s}}{i \tau} d \beta_{\ell}(s)\right.\right. \\
& \left.\left.\quad \times \theta^{2}(t) e^{-i t n^{\prime 3}} \varphi_{n^{\prime}, m}(t) \frac{e^{i \tau t}}{-i \tau} d \beta_{m}(t)\right)\left.d \tau\right|^{2}\right) \\
& =\mathbb{E}\left(\mid \sum_{m=0}^{\infty} \int_{\mathbb{R}} \sum_{\left|n^{\prime}\right| \sim 2^{n}} \sum_{\ell=0}^{\infty} \int_{-\infty}^{t} 2 \operatorname{Re}\left(\theta^{2}(s) e^{-i(t-s) n^{\prime 3}} \int_{|\tau| \sim 2^{k}} \frac{e^{i \tau(t-s)}}{\tau^{2}} d \tau\right.\right. \\
& \left.\left.\quad \times \theta^{2}(t)\right)\left.\varphi_{n^{\prime}, \ell}(s) d \beta_{\ell}(s) \varphi_{n^{\prime}, m}(t) d \beta_{m}(t)\right|^{2}\right) \\
& =\sum_{m=0}^{\infty} \int_{\mathbb{R}} \sum_{\mathbb{E} \mid} \sum_{\left|n^{\prime}\right| \sim 2^{n}} \sum_{\ell=0}^{\infty} \int_{-\infty}^{t} 2 \operatorname{Re}\left(\theta^{2}(s) \theta^{2}(t) e^{-i(t-s) n^{\prime 3}} \int_{|\tau| \sim 2^{k}} \frac{e^{i \tau(t-s)}}{\tau^{2}} d \tau\right) \\
& \quad \times\left.\varphi_{n^{\prime}, \ell}(s) d \beta_{\ell}(s) \varphi_{n^{\prime}, m}(t)\right|^{2} d t
\end{aligned}
$$


where we have used again the independence of the family $\left(\beta_{m}\right)_{m \in \mathbb{N}}$. Using now Cauchy-Schwarz inequality in $n^{\prime}$, the above term is bounded by

$$
\begin{aligned}
& \sum_{m=0}^{\infty} \int_{\mathbb{R}} \mathbb{E}\left(\sum _ { | n ^ { \prime } | \sim 2 ^ { n } } \left(\sum _ { \ell = 0 } ^ { \infty } \int _ { - \infty } ^ { t } 2 \operatorname { R e } \left(\theta^{2}(s) \theta^{2}(t) e^{-i(t-s) n^{\prime 3}}\right.\right.\right. \\
& \left.\left.\left.\quad \times \int_{|\tau| \sim 2^{k}} \frac{e^{i \tau(t-s)}}{\tau^{2}} d \tau\right) \varphi_{n^{\prime}, \ell}(s) d \beta_{\ell}(s)\right)^{2} \sum_{\left|n^{\prime}\right| \sim 2^{n}} \varphi_{n^{\prime}, m}^{2}(t)\right) d t \\
& \left.\leq \sup _{[0, t] \times \Omega} \sum_{\left|n^{\prime}\right| \sim 2^{n}} \sum_{m=0}^{\infty}\left|\varphi_{n^{\prime}, m}(t)\right|^{2}\right) \\
& \quad \times \int_{\mathbb{R}} \mathbb{E} \sum_{\left|n^{\prime}\right| \sim 2^{n}}\left(\sum_{\ell=0}^{\infty} \int_{-\infty}^{t} 2 \operatorname{Re}\left(\theta^{2}(s) \theta^{2}(t) e^{-i(t-s) n^{\prime 3}} \int_{|\tau| \sim 2^{k}} \frac{e^{i \tau(t-s)}}{\tau^{2}} d \tau\right)\right. \\
& \left.\quad \times \varphi_{n^{\prime}, \ell}(s) d \beta_{\ell}(s)\right)^{2} d t .
\end{aligned}
$$

Concerning the first term in the right hand side above, we have

$$
\begin{aligned}
& \sup _{[0, T] \times \Omega} \sum_{\left|n^{\prime}\right| \sim 2^{n}} \sum_{m=0}^{\infty}\left|\varphi_{n^{\prime}, m}(t)\right|^{2} \\
& \leq \sup _{[0, T] \times \Omega} \sum_{m=0}^{\infty} \sum_{\left|n^{\prime}\right| \sim 2^{n}}\left|\phi(t) e_{m}\left(n^{\prime}\right)\right|^{2} \\
& \leq \sup _{[0, T] \times \Omega} \sum_{m=0}^{\infty}\left|\Delta_{n} \phi(t) e_{m}\right|_{L^{2}(\mathbb{T})}^{2}=\left\|\Delta_{n} \phi(\cdot)\right\|_{L^{\infty}\left([0, T] \times \Omega ; L_{2}^{0,0}\right)}^{2}
\end{aligned}
$$

while the remaining term in $(2.5)$ is bounded above by

$$
\begin{aligned}
& \int_{\mathbb{R}} \sum_{\left|n^{\prime}\right| \sim 2^{n}} \sum_{\ell=0}^{\infty} \int_{-\infty}^{t} \theta^{4}(t) \theta^{4}(s)\left|\int_{|\tau| \sim 2^{k}} \frac{e^{i \tau(t-s)}}{\tau^{2}} d \tau\right|^{2} \mathbb{E}\left(\left|\varphi_{n^{\prime}, \ell}(s)\right|^{2}\right) d s d t \\
& \leq\left\|\Delta_{n} \phi(\cdot)\right\|_{L^{\infty}\left([0, T] \times \Omega ; L_{2}^{0,0}\right)}^{2} \int_{\mathbb{R}} \int_{-\infty}^{t} \theta^{4}(t) \theta^{4}(s)\left|\int_{|\tau| \sim 2^{k}} \frac{e^{i \tau(t-s)}}{\tau^{2}} d \tau\right|^{2} d s d t .
\end{aligned}
$$

We then notice that, by interpolation between the cases $\alpha=0$ and $\alpha=1$, for any $\alpha \in[0,1]$ there is a positive constant $C_{\alpha}$ such that

$$
\left|\int_{|\tau| \sim 2^{k}} \frac{e^{i \tau(t-s)}}{\tau^{2}} d \tau\right| \leq \frac{C_{\alpha}}{|t-s|^{\alpha}} 2^{-(1+\alpha) k} .
$$


Applying this with $\alpha=1 / 4$, we get that the second term in (2.5) is bounded above by

$$
\begin{aligned}
& C 2^{-\frac{5}{2} k}\left\|\Delta_{n} \phi(\cdot)\right\|_{L^{\infty}\left([0, T] \times \Omega ; L_{2}^{0,0}\right)}^{2} \int_{\mathbb{R}} \int_{-\infty}^{t} \frac{\theta^{4}(t) \theta^{4}(s)}{\sqrt{t-s}} d s d t \\
& \leq C(\theta) 2^{-\frac{5}{2} k}\left\|\Delta_{n} \phi(\cdot)\right\|_{L^{\infty}\left([0, T] \times \Omega ; L_{2}^{0,0}\right)^{\prime}}^{2}
\end{aligned}
$$

Collecting all these estimates from (2.4), we get

$$
\mathbb{E}\left(\left|\sum_{\left|n^{\prime}\right| \sim 2^{n}} \int_{|\tau| \sim 2^{k}} I_{n^{\prime}}^{1}(\tau) d \tau\right|^{2}\right) \leq C(\theta) 2^{-\frac{5}{2} k}\left\|\Delta_{n} \phi(\cdot)\right\|_{L^{\infty}\left([0, T] \times \Omega ; L_{2}^{0,0}\right)}^{4}
$$

and we deduce from this latest inequality that

$$
\begin{aligned}
& \mathbb{E}\left(\sum_{n=0}^{\infty} \sup _{k \in \mathbb{N}} 2^{k / 2}\left(\sum_{\left|n^{\prime}\right| \sim 2^{n}} \int_{|\tau| \sim 2^{k}} I_{n^{\prime}}^{1}(\tau) d \tau\right)^{1 / 2}\right) \\
& \leq \mathbb{E}\left(\sum_{n=0}^{\infty} \sum_{k=0}^{\infty} 2^{k / 2}\left(\sum_{\left|n^{\prime}\right| \sim 2^{n}} \int_{|\tau| \sim 2^{k}} I_{n^{\prime}}^{1}(\tau) d \tau\right)^{1 / 2}\right) \\
& \leq \sum_{n=0}^{\infty} \sum_{k=0}^{\infty} 2^{k / 2}\left[\mathbb{E}\left(\left(\sum_{\left|n^{\prime}\right| \sim 2^{n}} \int_{|\tau| \sim 2^{k}} I_{n^{\prime}}^{1}(\tau) d \tau\right)^{2}\right)\right]^{1 / 4} \\
& \leq C(\theta) \sum_{n=0}^{\infty}\left(\sum_{k=0}^{\infty} 2^{-\frac{k}{8}}\right)\left\|\Delta_{n} \phi(\cdot)\right\|_{L^{\infty}\left([0, T] \times \Omega ; L_{2}^{0,0}\right)}
\end{aligned}
$$

where we have used Hölder's inequality at the third line; this, together with (2.3), completes the proof of the estimate of $I$.

In this way, the first inequality in Proposition 2.1 is proved after an application of Lemma 1.1, with $\sigma^{\prime}<\sigma$. The second inequality follows from the obvious fact that

$$
\begin{aligned}
& \sum_{n=0}^{\infty}\left\|\Delta_{n} \phi(\cdot)\right\|_{L^{\infty}\left([0, T] \times \Omega ; L_{2}^{0, \sigma}\right)}^{2} \\
& =\sum_{n=0}^{\infty} 2^{\sigma n}\left\|\Delta_{n} \phi(\cdot)\right\|_{L^{\infty}\left([0, T] \times \Omega ; L_{2}^{0,0}\right)}^{2} \\
& \leq\left(\sum_{n=0}^{\infty} 2^{-2\left(s^{\prime}-\sigma\right) n}\right)^{1 / 2}\left(\sum_{n=0}^{\infty} 2^{s^{\prime} n}\left\|\Delta_{n} \phi(\cdot)\right\|_{L^{\infty}\left([0, T] \times \Omega ; L_{2}^{0,0}\right)}^{2}\right)^{1 / 2} \\
& \leq C\left(s^{\prime}, \sigma\right)\|\phi(\cdot)\|_{L^{\infty}\left([0, T] \times \Omega ; L_{2}^{0, s^{\prime}}\right)}
\end{aligned}
$$




\section{Bilinear estimates}

We now turn to prove some bilinear estimates which will allow us to handle the nonlinear term in equation (1.5). The next one is the crucial estimate.

Proposition 3.1. Let $-\frac{1}{2} \leq s \leq 0$ and $f \in X_{1,1}^{s, 1 / 2}, g \in X_{1, \infty}^{s, 1 / 2}$; then $\partial_{x}(f g) \in$ $X_{1,1}^{s,-1 / 2}$ and there is a constant $C>0$ such that

$$
\left|\partial_{x}(f g)\right|_{X_{1,1}^{s,-1 / 2}} \leq C|f|_{X_{1,1}^{s, 1 / 2}}|g|_{X_{1, \infty}^{s, 1 / 2}}
$$

Proof. Let $f$ and $g$ be as above; using a duality argument, it is sufficient, as usually, to prove that for some constant $C>0$, and for any function $h$ in $X_{\infty, \infty}^{-s, 1 / 2}$ - where $X_{\infty, \infty}^{-s, 1 / 2}$ is defined in an obvious way by modifying the definition of $X_{1,1}^{-s, 1 / 2}$ - we have

$$
\left|\left\langle\partial_{x}(f g), h\right\rangle\right| \leq C|f|_{X_{1,1}^{s, 1 / 2}}|g|_{X_{1, \infty}^{s, 1 / 2}}|h|_{X_{\infty, \infty}^{-s, 1 / 2}}
$$

Using Plancherel Theorem, one has

$\left|\left\langle\partial_{x}(f g), h\right\rangle\right|=\left|\sum_{n^{\prime} \neq 0} \sum_{\substack{n_{1}^{\prime} \neq 0 \\ n_{1}^{\prime} \neq n^{\prime}}} \int_{\tau \in \mathbb{R}} \int_{\tau_{1} \in \mathbb{R}} n^{\prime} \hat{\hat{h}}\left(\tau, n^{\prime}\right) \hat{g}\left(\tau_{1}, n_{1}^{\prime}\right) \hat{f}\left(\tau-\tau_{1}, n^{\prime}-n_{1}^{\prime}\right) d \tau_{1} d \tau\right|$.

We will denote $\sigma=\sigma\left(\tau, n^{\prime}\right)=\tau-n^{\prime 3}, \sigma_{1}=\sigma\left(\tau_{1}, n_{1}^{\prime}\right), \sigma_{2}=\sigma\left(\tau-\tau_{1}, n^{\prime}-\right.$ $\left.n_{1}^{\prime}\right)$. We also set $\hat{G}\left(\tau, n^{\prime}\right)=n^{\prime s}\langle\sigma\rangle^{1 / 2} \hat{g}\left(\tau, n^{\prime}\right), \hat{F}\left(\tau, n^{\prime}\right)=n^{\prime s}\langle\sigma\rangle^{1 / 2} \hat{f}\left(\tau, n^{\prime}\right)$ and $\hat{H}\left(\tau, n^{\prime}\right)=n^{\prime-s}\langle\sigma\rangle^{1 / 2} \hat{\hat{h}}\left(\tau, n^{\prime}\right)$, so that $F, G$ and $H$ lie respectively in $X_{1,1}^{0,0}, X_{1, \infty}^{0,0}$ and $X_{\infty, \infty}^{0,0}$. It suffices to prove that

$$
\begin{aligned}
& \sum_{n^{\prime} \neq 0} \sum_{\substack{n_{1}^{\prime} \neq 0 \\
n_{1}^{\prime} \neq n^{\prime}}} \int_{\tau \in \mathbb{R}} \int_{\tau_{1} \in \mathbb{R}} \frac{\left|n^{\prime}\right|^{1+s}\left|n_{1}^{\prime}\right|^{-s}\left|n^{\prime}-n_{1}^{\prime}\right|^{-s}\left|\hat{H}_{\tau, n^{\prime}}\right|\left|\hat{G}_{\tau_{1}, n_{1}^{\prime}}\right|\left|\hat{F}_{\tau-\tau_{1}, n^{\prime}-n_{1}^{\prime}}\right|}{\langle\sigma\rangle^{1 / 2}\left\langle\sigma_{1}\right\rangle^{1 / 2}\left\langle\sigma_{2}\right\rangle^{1 / 2}} d \tau_{1} d \tau \\
& \leq C|H|_{X_{\infty, \infty}^{0,0}}|G|_{X_{1, \infty}^{0,0}}|F|_{X_{1,1}^{0,0},}
\end{aligned}
$$

where we use $\hat{H}_{\tau, n^{\prime}}$ for $\hat{H}\left(\tau, n^{\prime}\right)$ and so on. We divide the region $\left(n^{\prime}, n_{1}^{\prime}, \tau, \tau_{1}\right) \in$ $(\mathbb{Z} \backslash\{0\})^{2} \times \mathbb{R}^{2}$ arising in the left hand side of (3.1) into three subregions:

$$
\begin{array}{rlrl}
(\text { Region I) } & \left\langle\sigma_{1}\right\rangle & =\max \left\{\langle\sigma\rangle,\left\langle\sigma_{1}\right\rangle,\left\langle\sigma_{2}\right\rangle\right\} \\
(\text { Region II }) & \langle\sigma\rangle & =\max \left\{\langle\sigma\rangle,\left\langle\sigma_{1}\right\rangle,\left\langle\sigma_{2}\right\rangle\right\} \\
\text { (Region III) } & \left\langle\sigma_{2}\right\rangle=\max \left\{\langle\sigma\rangle,\left\langle\sigma_{1}\right\rangle,\left\langle\sigma_{2}\right\rangle\right\}
\end{array}
$$


and we estimate separately the contributions of each of these regions to the left hand side of (3.1).

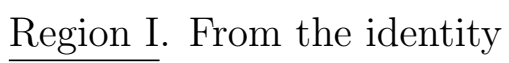

$$
3\left|n^{\prime}\right|\left|n_{1}^{\prime}\right|\left|n^{\prime}-n_{1}^{\prime}\right|=\left|\tau-n^{\prime 3}-\left(\tau_{1}-n_{1}^{\prime 3}\right)-\left(\left(\tau-\tau_{1}\right)-\left(n^{\prime}-n_{1}^{\prime}\right)^{3}\right)\right|
$$

we get as usually that in region I,

$$
\frac{1}{2}\left|n^{\prime}\right|^{2} \leq\left|n^{\prime}\right|\left|n_{1}^{\prime}\right|\left|n^{\prime}-n_{1}^{\prime}\right| \leq\left\langle\sigma_{1}\right\rangle
$$

so that for any $s \in\left[-\frac{1}{2}, 0\right]$,

$$
\left|n^{\prime}\right|^{1+s}\left|n_{1}^{\prime}\right|^{-s}\left|n^{\prime}-n_{1}^{\prime}\right|^{-s} \leq C\left\langle\sigma_{1}\right\rangle^{1 / 2}
$$

Hence, it is sufficient to prove that the contribution of region I to

$$
I=\sum_{n^{\prime} \neq 0} \sum_{\substack{n_{1}^{\prime} \neq 0, n_{1}^{\prime} \neq n^{\prime}}} \int_{\tau \in \mathbb{R}} \int_{\tau_{1} \in \mathbb{R}} \frac{\left|\hat{H}_{\tau, n^{\prime}}\right|\left|\hat{G}_{\tau_{1}, n_{1}^{\prime}}\right|\left|\hat{F}_{\tau-\tau_{1}, n^{\prime}-n_{1}^{\prime}}\right|}{\langle\sigma\rangle^{1 / 2}\left\langle\sigma_{2}\right\rangle^{1 / 2}} d \tau_{1} d \tau
$$

is bounded above by $C|H|_{X_{\infty, \infty}^{0,0}}|G|_{X_{1, \infty}^{0,0}}|F|_{X_{1,1}^{0,0}}$. Again, we will divide region I into several subregions.

Region $I$-a. We consider here the subregion for which $\langle\sigma\rangle \geq \frac{1}{4} n^{\prime 2}$.

We then estimate the contribution of this region to I; it is bounded above by its contribution to

$$
\sum_{n, n_{1} \in \mathbb{N}} \sum_{k, k_{1} \in \mathbb{N}} \sum_{\substack{\left|n^{\prime}\right| \sim 2^{n} \\\left|n_{1}^{\prime}\right| \sim 2^{n_{1}} \\ n^{\prime} \neq n_{1}^{\prime}}} \int_{|\tau| \sim 2^{k}} \int_{\left|\tau_{1}\right| \sim 2^{k_{1}}} \frac{\left|\hat{H}_{\tau, n^{\prime}}\right|\left|\hat{G}_{\tau_{1}, n_{1}^{\prime}}\right|\left|\hat{F}_{\tau-\tau_{1}, n^{\prime}-n_{1}^{\prime}}\right|}{\langle\sigma\rangle^{1 / 2}\left\langle\sigma_{2}\right\rangle^{1 / 2}} d \tau_{1} d \tau
$$

with the convention that for $k=0,|\tau| \sim 2^{k}$ means $|\tau| \leq 2$. This latest term is bounded above, using Cauchy-Schwarz inequality, by

$$
\begin{aligned}
& \sum_{n, n_{1} \in \mathbb{N}} \sum_{k, k_{1} \in \mathbb{N}}\left(\sum_{\left|n_{1}^{\prime}\right| \sim 2^{n_{1}}} \int_{\left|\tau_{1}\right| \sim 2^{k_{1}}}\left|\hat{G}_{\tau_{1}, n_{1}^{\prime}}\right|^{2} d \tau_{1}\right)^{1 / 2} \\
& \times\left(\sum_{\left|n_{1}^{\prime}\right| \sim 2^{n_{1}}} \int_{\left|\tau_{1}\right| \sim 2^{k_{1}}}\left(\sum_{\left|n^{\prime}\right| \sim 2^{n}} \int_{|\tau| \sim 2^{k}} \frac{\left|\hat{H}_{\tau, n^{\prime}}\right| \mid \hat{F}_{\tau-\tau_{1}, n^{\prime}-n_{1}^{\prime} \mid}}{\langle\sigma\rangle^{1 / 2}\left\langle\sigma_{2}\right\rangle^{1 / 2}} d \tau\right)^{2} d \tau_{1}\right)^{1 / 2} .
\end{aligned}
$$


Now, we use the fact that in region I-a, we have for $\varepsilon>0$ small, which will be chosen more precisely later,

$$
\frac{1}{\left\langle\sigma\left(\tau, n^{\prime}\right)\right\rangle^{1 / 2}} \leq C\left|n^{\prime}\right|^{-\varepsilon} \frac{1}{\left\langle\sigma\left(\tau, n^{\prime}\right\rangle\right)^{1 / 2-\varepsilon / 2}}
$$

and using Cauchy-Schwarz inequality in $\left(\tau, n^{\prime}\right)$ in $(3.3)$, it is bounded above by

$$
\begin{aligned}
& C \sum_{n, n_{1} \in \mathbb{N}} \sum_{k, k_{1} \in \mathbb{N}}\left(\sum_{\left|n_{1}^{\prime}\right| \sim 2^{n_{1}}} \int_{\left|\tau_{1}\right| \sim 2^{k_{1}}}\left|\hat{G}_{\tau_{1}, n_{1}^{\prime}}\right|^{2} d \tau_{1}\right)^{1 / 2} \\
& \times\left[\sum_{\left|n_{1}^{\prime}\right| \sim 2^{n_{1}}} \int_{\left|\tau_{1}\right| \sim 2^{k_{1}}}\left(\sum_{\left|n^{\prime}\right| \sim 2^{n}} \int_{|\tau| \sim 2^{k}}\left|n^{\prime}\right|^{-2 \varepsilon}\left\langle\sigma_{\tau, n^{\prime}}\right\rangle^{-\varepsilon}\left|\hat{H}_{\tau, n^{\prime}}\right|^{2}\left|\hat{F}_{\tau-\tau_{1}, n^{\prime}-n_{1}^{\prime}}\right|^{2} d \tau\right)\right. \\
& \left.\times\left(\sum_{\left|n^{\prime}\right| \sim 2^{n}} \int_{\mid \tau \sim 2^{k}} \frac{d \tau}{\left\langle\sigma_{\tau, n^{\prime}}\right\rangle^{1-2 \varepsilon}\left\langle\sigma_{\tau-\tau_{1}, n^{\prime}-n_{1}^{\prime}}\right\rangle}\right) d \tau_{1}\right]^{1 / 2} \\
& \leq C \sum_{n_{1} \in \mathbb{N}}\left[\sup _{k_{1} \in \mathbb{N}}\left(\sum_{\left|n_{1}^{\prime}\right| \sim 2^{n_{1}}} \int_{\left|\tau_{1}\right| \sim 2^{k_{1}}}\left|\hat{G}_{\tau_{1}, n_{1}^{\prime}}\right|^{2} d \tau_{1}\right)^{1 / 2}\right. \\
& \times \sup _{k_{1}, n, k \in \mathbb{N}} \sup _{\left|n_{1}^{\prime}\right| \sim 2^{n_{1}}} \sup _{\left|\tau_{1}\right| \sim 2^{k_{1}}}\left(\sum_{\left|n^{\prime}\right| \sim 2^{n}} \int_{|\tau| \sim 2^{k}} \frac{d \tau}{\left\langle\sigma_{\tau, n^{\prime}}\right\rangle^{1-2 \varepsilon}\left\langle\sigma_{\tau-\tau_{1}, n^{\prime}-n_{1}^{\prime}}\right\rangle}\right)^{1 / 2} \\
& \times \sum_{k_{1}, n, k \in \mathbb{N}}\left(\sum_{\left|n_{1}^{\prime}\right| \sim 2^{n_{1}}} \int_{\left|\tau_{1}\right| \sim 2^{k_{1}}} \sum_{\left|n^{\prime}\right| \sim 2^{n}} \int_{|\tau| \sim 2^{k}}\left|n^{\prime}\right|^{-2 \varepsilon}\left\langle\sigma_{\tau, n^{\prime}}\right\rangle^{-\varepsilon}\left|\hat{H}_{\tau, n^{\prime}}\right|^{2}\right. \\
& \left.\left.\times\left|\hat{F}_{\tau-\tau_{1}, n^{\prime}-n_{1}^{\prime}}\right|^{2} d \tau d \tau_{1}\right)^{1 / 2}\right] \text {. }
\end{aligned}
$$

But now, the fact that

$$
\int_{-\infty}^{+\infty} \frac{d \theta}{(1+|\theta|)^{1-2 \varepsilon}(1+|\theta-a|)} \leq \frac{C}{(1+|a|)^{1-4 \varepsilon}}
$$

for $a \in \mathbb{R}$, and the proof of Lemma 5.1 in [15] show that there is a constant $C>0$ such that

$$
\begin{aligned}
& \sup _{n_{1} \in \mathbb{Z}^{*}} \sup _{\tau_{1} \in \mathbb{R}} \sup _{n, k \in \mathbb{N}}\left(\sum_{\substack{\left|n^{\prime}\right| \sim 2^{n}\\
}} \int_{|\tau| \sim 2^{k}} \frac{d \tau}{\left\langle\sigma_{\tau, n^{\prime}}\right\rangle^{1-2 \varepsilon}\left\langle\sigma_{\tau-\tau_{1}, n^{\prime}-n_{1}}\right\rangle}\right) \\
& \leq \sup _{n_{1} \in \mathbb{Z}^{*}} \sup _{\tau_{1} \in \mathbb{R}}\left(\sum_{\substack{n \in \mathbb{Z} \backslash\{0\} \\
n \neq n_{1}}} \int_{\tau \in \mathbb{R}} \frac{d \tau}{\left\langle\sigma_{\tau, n}\right\rangle^{1-2 \varepsilon}\left\langle\sigma_{\tau-\tau_{1}, n-n_{1}}\right\rangle}\right) \\
& \leq C,
\end{aligned}
$$


for any $\varepsilon>0$ such that $1-4 \varepsilon \geq 3 / 4$, i.e. for any $\varepsilon \leq 1 / 16$. On the other hand the last line in (3.4) is bounded above by

$$
\begin{aligned}
& \sum_{\substack{n, n_{1} \in \mathbb{N} \\
k, k_{1} \in \mathbb{N}}}\left[\left(\sum_{\left|n^{\prime}\right| \sim 2^{n}} \int_{|\tau| \sim 2^{k}}\left|n^{\prime}\right|^{-\varepsilon}\langle\sigma\rangle^{-\varepsilon / 2}\left|\hat{H}_{\tau, n^{\prime}}\right|^{2} d \tau\right)^{1 / 2}\right. \\
& \left.\quad \times \sup _{\left|n^{\prime}\right| \sim 2^{n}} \sup _{|\tau| \sim 2^{k}}\left(\sum_{\left|n_{1}^{\prime}\right| \sim 2^{n_{1}}} \int_{\left|\tau_{1}\right| \sim 2^{k_{1}}}\left|n^{\prime}\right|^{-\varepsilon}\langle\sigma\rangle^{-\varepsilon / 2}\left|\hat{F}_{\tau-\tau_{1}, n^{\prime}-n_{1}^{\prime}}\right|^{2} d \tau_{1}\right)^{1 / 2}\right] \\
& \leq \sup _{n, k \in \mathbb{N}} \sum_{n_{1}, k_{1} \in \mathbb{N}} \sup _{\substack{\left|n^{\prime}\right| \sim 2^{n} \\
|\tau| \sim 2^{k}}}\left(\sum_{\left|n_{1}^{\prime}\right| \sim 2^{n_{1}}} \int_{\left|\tau_{1}\right| \sim 2^{k_{1}}}\left|n^{\prime}\right|^{-\varepsilon}\langle\sigma\rangle^{-\varepsilon / 2} \mid \hat{F}_{\left.\tau-\tau_{1}, n^{\prime}-\left.n_{1}^{\prime}\right|^{2} d \tau_{1}\right)^{1 / 2}}\right. \\
& \quad \times \sum_{n, k \in \mathbb{N}}\left(\sum_{\substack{\left|n^{\prime}\right| \sim 2^{n} \\
\leq}} C_{|\tau| c \mid \sim 2^{k}}\left|n^{\prime}\right|^{-\varepsilon}\langle\sigma\rangle^{-\varepsilon / 2}\left|\hat{H}_{\tau, n^{\prime}}\right|^{2} d \tau\right)^{1 / 2} \\
& \times \sup _{n, k \in \mathbb{N}} \sum_{n_{1}, k_{1} \in \mathbb{N}}\left(\sup _{\substack{\left|n^{\prime}\right| \sim 2^{n} \\
|\tau| \sim 2^{k}}}\left(\sum_{\left|n_{1}^{\prime}\right| \sim 2^{n_{1}}} \int_{\left|t_{1}\right| \sim 2^{k_{1}}}\left|n^{\prime}\right|^{-\varepsilon}\langle\sigma\rangle^{-\varepsilon / 2}\left|\hat{F}_{\tau-\tau_{1}, n^{\prime}-n_{1}^{\prime}}\right|^{2} d \tau_{1}\right)^{1 / 2}\right) .
\end{aligned}
$$

One may then notice that if $\left|n_{1}^{\prime}\right| \geq 4\left|n^{\prime}\right|$, then $\left|n^{\prime}-n_{1}^{\prime}\right| \sim 2^{n_{1}}$ and if $\left|\tau_{1}\right| \geq 4|\tau|$, then $\left|\tau-\tau_{1}\right| \sim 2^{k_{1}}$, so that for any $n, k \in \mathbb{N}$,

$$
\begin{aligned}
& \sum_{n_{1}, k_{1} \in \mathbb{N}} \sup _{\substack{\left|n^{\prime}\right| \sim 2^{n} \\
|\tau| \sim 2^{k}}}\left(\sum_{\substack{\left|n_{1}^{\prime}\right| 2^{n_{1}} \\
\left|n_{1}^{\prime}\right| \geq 4\left|n^{\prime}\right|}} \int_{\substack{\left|\tau_{1}\right| \sim 2^{k_{1}} \\
\left|\tau_{1}\right| \geq 4|\tau|}}\left|n^{\prime}\right|^{-\varepsilon}\langle\sigma\rangle^{-\varepsilon / 2}\left|\hat{F}_{\tau-\tau_{1}, n^{\prime}-n_{1}^{\prime}}\right|^{2} d \tau_{1}\right)^{1 / 2} \\
& \leq C \sum_{n_{1}, k_{1} \in \mathbb{N}}\left(\sum_{\left|n_{1}^{\prime}\right| \sim 2^{n_{1}}} \int_{\left|\tau_{1}\right| \sim 2^{k_{1}}}\left|\hat{F}_{\tau_{1}, n_{1}^{\prime}}\right|^{2} d \tau_{1}\right)^{1 / 2} \\
& \leq C|F|_{X_{1,1}^{0,0}},
\end{aligned}
$$


while if $\left|n_{1}^{\prime}\right| \leq 4\left|n^{\prime}\right|$ (with still $\left.\left|\tau_{1}\right| \geq 4|\tau|\right)$ then $\left|n^{\prime}\right|^{-\varepsilon} \leq C\left|n_{1}^{\prime}\right|^{-\varepsilon}$ and $\forall n, k, \in \mathbb{N}$,

$$
\begin{aligned}
& \sum_{n_{1}, k_{1} \in \mathbb{N}} \sup _{\substack{\left|n^{\prime}\right| \sim 2^{n} \\
|\tau| \sim 2^{k}}}\left(\sum_{\substack{\left|n_{1}^{\prime}\right| \sim 2^{n_{1}} \\
\left|n_{1}^{\prime}\right| \leq 4\left|n^{\prime}\right|}} \int_{\substack{\left|\tau_{1}\right| \sim 2^{k_{1}} \\
\left|\tau_{1}\right| \geq 4|\tau|}}\left|n^{\prime}\right|^{-\varepsilon}\langle\sigma\rangle^{-\varepsilon / 2}\left|\hat{F}_{\tau-\tau_{1}, n^{\prime}-n_{1}^{\prime}}\right|^{2} d \tau_{1}\right)^{1 / 2} \\
& \leq C \sum_{n_{1} \in \mathbb{N}}\left(\sup _{\left|n_{1}^{\prime}\right| \sim 2^{n_{1}}}\left|n_{1}^{\prime}\right|^{-\varepsilon}\right) \sum_{k_{1} \in \mathbb{N}} \sup _{\left|n^{\prime}\right| \sim 2^{n}}\left(\sum_{\left|n_{1}^{\prime}\right| \sim 2^{n_{1}}} \int_{\left|\tau_{1}\right| \sim 2^{k_{1}}}\left|\hat{F}_{\tau_{1}, n^{\prime}-n_{1}^{\prime}}\right|^{2} d \tau_{1}\right)^{1 / 2} \\
& \leq C_{\varepsilon} \sum_{k_{1} \in \mathbb{N}}\left(\sum_{\ell \in \mathbb{Z}} \int_{\left|\tau_{1}\right| \sim 2^{k_{1}}}\left|\hat{F}_{\tau, \ell}\right|^{2} d \tau\right)^{1 / 2} \\
& \leq C_{\varepsilon} \sum_{k_{1} \in \mathbb{N}} \sum_{\ell \in \mathbb{N}}\left(\sum_{\left|\ell^{\prime}\right| \sim 2^{\ell}} \int_{\left|\tau_{1}\right| \sim 2^{k_{1}}}\left|\hat{F}_{\tau, \ell^{\prime}}\right|^{2} d \tau\right)^{1 / 2} \\
& \leq C_{\varepsilon}|F|_{X_{1,1}^{0,0}} .
\end{aligned}
$$

The cases for which $\left|\tau_{1}\right| \leq 4|\tau|$ are treated in the same way as the latest case above, using that in this case, $\langle\sigma\rangle^{-\varepsilon} \leq C\left\langle\tau_{1}-n^{\prime 3}\right\rangle^{-\varepsilon}$ so that the sum over $k_{1}$ converges.

It follows from these estimates that (3.4) is bounded above by

$$
C|G|_{X_{\infty}^{0,0}, \infty}|H|_{X_{\infty}^{0,0}}|F|_{X_{1,1}^{0,0}} \leq C|G|_{X_{1, \infty}^{0,0}}|H|_{X_{\infty}^{0,0}, \infty}|F|_{X_{1,1}^{0,0}}
$$

and this achieves the estimate of the contribution of Region I-a.

Region I-b. Assume here that $\left\langle\sigma_{2}\right\rangle \geq \frac{1}{4}{n^{\prime}}^{2}$.

We may then proceed as in Region I-a, by noticing that here, we have for $\varepsilon>0$ small,

$$
\frac{1}{\langle\sigma\rangle^{1 / 2}\left\langle\sigma_{2}\right\rangle^{1 / 2}} \leq C_{\varepsilon}\left|n^{\prime}\right|^{-\varepsilon}\langle\sigma\rangle^{-\varepsilon / 2} \frac{1}{\langle\sigma\rangle^{1 / 2-\varepsilon / 2}\left\langle\sigma_{2}\right\rangle^{1 / 2-\varepsilon / 2}}
$$

and that

$$
\sup _{\substack{n, k \in \mathbb{N} \\ n_{1}^{\prime} \in \mathbb{Z} \backslash\{0\} \\ \tau_{1} \in \mathbb{R}}}\left(\sum_{\left|n^{\prime}\right| \sim 2^{n}} \int_{|\tau| \sim 2^{k}} \frac{d \tau}{\left\langle\sigma\left(\tau, n^{\prime}\right)\right\rangle^{1-\varepsilon}\left\langle\sigma\left(\tau-\tau_{1}, n^{\prime}-n_{1}^{\prime}\right)\right\rangle^{1-\varepsilon}}\right)<+\infty
$$

for any $\varepsilon \leq 1 / 8$.

Region I-c. We consider now the region where $\langle\sigma\rangle \leq \frac{1}{4} n^{\prime 2}$ and $\left\langle\sigma_{2}\right\rangle \leq \frac{1}{4} n^{\prime 2}$.

The contribution of this region to $I$ will be the most difficult to estimate. Again, we use in (3.2) Cauchy-Schwarz inequality in $\left(\tau, n^{\prime}\right)$, to bound the 
contribution of region I-c to (3.2) by its contribution to

$$
\begin{aligned}
& \sum_{n, n_{1} \in \mathbb{N}} \sum_{k, k_{1} \in \mathbb{N}}\left(\sum_{\left|n_{1}^{\prime}\right| \sim 2^{n_{1}}} \int_{\left|\tau_{1}\right| \sim 2^{k_{1}}}\left|\hat{G}_{\tau_{1}, n_{1}^{\prime}}\right|^{2} d \tau_{1}\right)^{1 / 2} \\
& \times\left[\sum_{\left|n_{1}^{\prime}\right| \sim 2^{n_{1}}} \int_{\left|\tau_{1}\right| \sim 2^{k_{1}}}\left(\sum_{\left|n^{\prime}\right| \sim 2^{n}} \int_{|\tau| \sim 2^{k}}\left|\hat{H}_{\tau, n^{\prime}}\right|^{2}\left|\hat{F}_{\tau-\tau_{1}, n^{\prime}-n_{1}^{\prime}}\right|^{2} d \tau\right)\right. \\
& \left.\times\left(\sum_{\left|n^{\prime}\right| \sim 2^{n}} \int_{|\tau| \sim 2^{k}} \frac{d \tau}{\langle\sigma\rangle\left\langle\sigma_{2}\right\rangle}\right)\right]^{1 / 2} \\
& \leq \sum_{n_{1} \in \mathbb{N}}\left[\sup _{k_{1} \in \mathbb{N}}\left(\sum_{\left|n_{1}^{\prime}\right| \sim 2^{n_{1}}} \int_{\left|\tau_{1}\right| \sim 2^{k_{1}}}\left|\hat{G}_{\tau_{1}, n_{1}^{\prime}}\right|^{2} d \tau_{1}\right)^{1 / 2}\right. \\
& \times \sum_{n \in \mathbb{N}} \sum_{k_{1} \in \mathbb{N}} \sum_{k \in \mathbb{N}}\left\{\sup _{\left|n_{1}^{\prime}\right| \sim 2^{n_{1}}} \sup _{\left|\tau_{1}\right| \sim 2^{k_{1}}}\left(\sum_{\left|n^{\prime}\right| \sim 2^{n}} \int_{|\tau| \sim 2^{k}} \frac{d \tau}{\langle\sigma\rangle\left\langle\sigma_{2}\right\rangle}\right)^{1 / 2}\right. \\
& \left.\left.\times\left(\sum_{\left|n_{1}^{\prime}\right| \sim 2^{n_{1}}} \int_{\left|\tau_{1}\right| \sim 2^{k_{1}}} \sum_{\left|n^{\prime}\right| \sim 2^{n}} \int_{|\tau| \sim 2^{k}}\left|\hat{H}_{\tau, n^{\prime}}\right|^{2}\left|\hat{F}_{\tau-\tau_{1}, n^{\prime}-n_{1}^{\prime}}\right|^{2} d \tau d \tau_{1}\right)^{1 / 2}\right\}\right] \\
& \leq \sum_{n_{1} \in \mathbb{N}}\left[\sup _{k_{1} \in \mathbb{N}}\left(\sum_{\left|n_{1}^{\prime}\right| \sim 2^{n_{1}}} \int_{\left|\tau_{1}\right| \sim 2^{k_{1}}}\left|\hat{G}_{\tau_{1}, n_{1}^{\prime}}\right|^{2} d \tau_{1}\right)^{1 / 2}\right. \\
& \times \sum_{n \in \mathbb{N}} \sum_{k_{1} \in \mathbb{N}}\left\{\sum_{k \in \mathbb{N}} \sup _{\left|n_{1}^{\prime}\right| \sim 2^{n_{1}}} \sup _{\left|\tau_{1}\right| \sim 2^{k_{1}}}\left(\sum_{\left|n^{\prime}\right| \sim 2^{n}} \int_{|\tau| \sim 2^{k}} \frac{d \tau}{\langle\sigma\rangle\left\langle\sigma_{2}\right\rangle}\right)^{1 / 2}\right. \\
& \left.\left.\times \sup _{k \in \mathbb{N}}\left(\sum_{\left|n_{1}^{\prime}\right| \sim 2^{n_{1}}} \int_{\left|\tau_{1}\right| \sim 2^{k_{1}}} \sum_{\left|n^{\prime}\right| \sim 2^{n}} \int_{|\tau| \sim 2^{k}}\left|\hat{H}_{\tau, n^{\prime}}\right|^{2}\left|\hat{F}_{\tau-\tau_{1}, n^{\prime}-n_{1}^{\prime}}\right|^{2} d \tau d \tau_{1}\right)^{1 / 2}\right\}\right]
\end{aligned}
$$

and using Cauchy-Schwarz inequality in $n$, this is bounded above by

$$
\begin{aligned}
\sum_{n_{1} \in \mathbb{N}}\{ & \sup _{k_{1} \in \mathbb{N}}\left(\sum_{\left|n_{1}^{\prime}\right| \sim 2^{n_{1}}} \int_{\left|\tau_{1}\right| \sim 2^{k_{1}}}\left|\hat{G}_{\tau_{1}, n_{1}^{\prime}}\right|^{2} d \tau_{1}\right)^{1 / 2} \\
& \times \sum_{k_{1} \in \mathbb{N}}\left[\left(\sum_{n \in \mathbb{N}}\left(\sum_{k \in \mathbb{N}} B\left(n_{1}, k_{1}, n, k\right)\right)^{2}\right)^{1 / 2}\right. \\
& \left.\left.\times\left(\sum_{n \in \mathbb{N}} \sup _{k \in \mathbb{N}} \sum_{\left|n_{1}^{\prime}\right| \sim 2^{n_{1}}} \int_{\left|\tau_{1}\right| \sim 2^{k_{1}}} \sum_{\left|n^{\prime}\right| \sim 2^{n}} \int_{|\tau| \sim 2^{k}}\left|\hat{H}_{\tau, n^{\prime}}\right|^{2}\left|\hat{F}_{\tau-\tau_{1}, n^{\prime}-n_{1}^{\prime}}\right|^{2} d \tau d \tau_{1}\right)^{1 / 2}\right]\right\}
\end{aligned}
$$


with

$$
B\left(n_{1}, k_{1}, n, k\right)=\sup _{\substack{\left|n_{1}^{\prime}\right| \sim 2^{n_{1}} \\\left|\tau_{1}\right| \sim 2^{k_{1}}}}\left(\sum_{\left.\right|_{n^{\prime} \mid \sim 2^{n}}} \int_{|\tau| \sim 2^{k}} \frac{d \tau}{\langle\sigma\rangle\left\langle\sigma_{2}\right\rangle}\right)^{1 / 2} .
$$

We will then make use of the following lemma.

Lemma 3.1. Let $N$ be an integer, $k_{0}$ a function of $\left(n_{1}, k_{1}, n\right) \in \mathbb{N}^{3}$ with values in $\mathbb{N}$, and $n_{0}$ a function of $\left(n_{1}, k_{1}\right) \in \mathbb{N}^{2}$ with values in $\mathbb{N}$. Denote by $A\left(N, n_{1}, k_{1}\right)$ the region in $\mathbb{N}^{2}$ given by

$$
\begin{aligned}
A\left(N, n_{1}, k_{1}\right)=\left\{(n, k) \in \mathbb{N}^{2},\right. & k_{0}\left(n_{1}, k_{1}, n\right) \leq k \leq k_{0}\left(n_{1}, k_{1}, n\right)+N, \\
& \left.n_{0}\left(n_{1}, k_{1}\right) \leq n \leq n_{0}\left(n_{1}, k_{1}\right)+N\right\} .
\end{aligned}
$$

Then there is a constant $C(N)$ depending only on $N$ such that

$$
\sup _{n_{1}, k_{1} \in \mathbb{N}} \sum_{(n, k) \in A\left(N, n_{1}, k_{1}\right)} B\left(n_{1}, k_{1}, n, k\right) \leq C(N),
$$

where $B\left(n_{1}, k_{1}, n, k\right)$ is defined by (3.6).

Proof of Lemma 3.1. It follows easily from Lemma 5.1 in [15], since

$$
\begin{aligned}
& \sup _{k_{1}, n_{1} \in \mathbb{N}} \sum_{\substack{(n, k) \\
\in A\left(N, n_{1}, k_{1}\right)}} B\left(n_{1}, k_{1}, n, k\right) \\
& \leq N^{2} \sup _{k_{1}, n_{1} \in \mathbb{N} N, \sup _{n \in \mathbb{N}} B\left(n_{1}, k_{1}, n, k\right)} \\
& \leq N^{2} \sup _{k_{1}, n_{1} \in \mathbb{N}}\left(\sum_{\substack{n \in \mathbb{Z} \backslash\{0\} \\
n \neq n_{1}}} \int_{\mathbb{R}} \frac{d \tau}{\langle\sigma(\tau, n)\rangle\left\langle\sigma\left(\tau-\tau_{1}, n-n_{1}\right)\right\rangle}\right)^{1 / 2} \\
& <+\infty
\end{aligned}
$$

by Lemma 5.1 in [15].

Now, in order to apply Lemma 3.1, we need to show that region I-c is embedded in a region of the form

$$
\left\{\left(n, k, n_{1}, k_{1}\right) \in \mathbb{N}^{4},(n, k) \in A\left(N, n_{1}, k_{1}\right)\right\}
$$

for some $N$ and for some functions $n_{0}\left(n_{1}, k_{1}\right)$ and $k_{0}\left(n_{1}, k_{1}, n\right)$.

Note that we have in region I-c :

$$
\left|\tau-n^{\prime 3}\right| \leq\langle\sigma(\tau, n)\rangle \leq \frac{1}{4} n^{\prime 2} \leq \frac{1}{4}\left|n^{\prime}\right|^{3}
$$


hence

$$
\frac{3}{4}\left|n^{\prime}\right|^{3} \leq|\tau| \leq \frac{5}{4}\left|n^{\prime}\right|^{3}
$$

and the property $3 n-4 \leq k \leq 3 n+4$ follows easily. Hence, to prove the preceding result, we only have to find $n_{0}\left(n_{1}, k_{1}\right)$ and $N$ such that for any $\left(n, k, n_{1}, k_{1}\right)$ in region I-c,

$$
n_{0}\left(n_{1}, k_{1}\right) \leq n \leq n_{0}\left(n_{1}, k_{1}\right)+N .
$$

In order to prove this fact, we again use a partition of region I-c into three subregions.

- Region I-c-1: $2^{-12}\left|n_{1}^{\prime}\right| \leq\left|n^{\prime}\right| \leq 2^{12}\left|n_{1}^{\prime}\right|$.

In this region, we obviously have the result with $n_{0}\left(n_{1}, k_{1}\right)=n_{1}-4$.

- Region I-c-2 : $\left|n^{\prime}\right| \leq 2^{-12}\left|n_{1}^{\prime}\right|$.

We recall that

$$
\left|\sigma-\sigma_{1}-\sigma_{2}\right|=3\left|n^{\prime}\right|\left|n_{1}^{\prime}\right|\left|n^{\prime}-n_{1}^{\prime}\right|
$$

from which it follows that (since $\left\langle\sigma_{1}\right\rangle$ is dominant)

$$
\left|n_{1}^{\prime}\right|\left|n^{\prime}\right|\left|n^{\prime}-n_{1}^{\prime}\right| \leq\left\langle\sigma_{1}\right\rangle \leq 3\left|n_{1}^{\prime}\right|\left|n^{\prime}\right|\left|n^{\prime}-n_{1}^{\prime}\right|+\langle\sigma\rangle+\left\langle\sigma_{2}\right\rangle ;
$$

using the fact that $\left|n^{\prime}\right| \leq \frac{1}{2}\left|n_{1}^{\prime}\right|$ and that $\langle\sigma\rangle \leq \frac{1}{4}\left|n^{\prime}\right|^{2}$ and $\left\langle\sigma_{2}\right\rangle \leq \frac{1}{4}\left|n^{\prime}\right|^{2}$, we easily get from the preceding inequality

$$
\frac{1}{2}\left|n_{1}^{\prime}\right|^{2}\left|n^{\prime}\right| \leq\left\langle\sigma_{1}\right\rangle \leq 5\left|n_{1}^{\prime}\right|^{2}\left|n^{\prime}\right|
$$

and the property follows easily with

$$
n_{0}\left(n_{1}, k_{1}\right)=\frac{\ln \left|2^{k_{1}}-2^{3 n_{1}}\right|}{\ln 2}-\frac{\ln 5}{\ln 2}-2 n_{1} .
$$

- Region I-c-3: $\left|n^{\prime}\right| \geq 2^{12}\left|n_{1}^{\prime}\right|$.

We infer here, from the inequality

$$
\left|n^{\prime}\right|\left|n_{1}^{\prime}\right|\left|n^{\prime}-n_{1}^{\prime}\right| \leq\left\langle\sigma_{1}\right\rangle \leq 3\left|n^{\prime}\right|\left|n_{1}^{\prime}\right|\left|n^{\prime}-n_{1}^{\prime}\right|+\langle\sigma\rangle+\left\langle\sigma_{2}\right\rangle
$$

that

$$
\frac{1}{2}\left|n^{\prime}\right|^{2}\left|n_{1}^{\prime}\right| \leq\left\langle\sigma_{1}\right\rangle \leq 5\left|n^{\prime}\right|^{2}\left|n_{1}^{\prime}\right|
$$

and we conclude as in the preceding case. 
Now, going back to (3.5), we may use Lemma 3.1 to show that the contribution of region I-c to

$$
\begin{aligned}
& \sup _{n_{1}, k_{1} \in \mathbb{N}}\left(\sum_{n \in \mathbb{N}}\left(\sum_{k \in \mathbb{N}} B\left(n_{1}, k_{1}, n, k\right)\right)^{2}\right)^{1 / 2} \\
& \leq \sup _{n_{1}, k_{1} \in \mathbb{N}} \sum_{n, k \in \mathbb{N}} B\left(n_{1}, k_{1}, n, k\right)
\end{aligned}
$$

is bounded above by an absolute constant.

Hence, each of the contributions of Regions I-c-1, I-c-2 and I-c-3 to (3.5) is bounded above by

$$
\begin{aligned}
& C \sum_{n_{1} \in \mathbb{N}}\left\{\sup _{k_{1} \in \mathbb{N}}\left(\sum_{\left|n_{1}^{\prime}\right| \sim 2^{n_{1}}} \int_{\left|\tau_{1}\right| \sim 2^{k_{1}}}\left|\hat{G}_{\tau_{1}, n_{1}}\right|^{2} d \tau_{1}\right)^{1 / 2}\right. \\
& \times \sum_{k_{1} \in \mathbb{N}}\left[\sum_{n=n_{0}\left(n_{1}, k_{1}\right)}^{n_{0}+N} \sup _{k_{0}(n) \leq k \leq k_{0}(n)+N} \sum_{\left|n_{1}^{\prime}\right| \sim 2^{n_{1}}} \int_{\left|\tau_{1}\right| \sim 2^{k_{1}}} \sum_{\left|n^{\prime}\right| \sim 2^{n}} \int_{|\tau| \sim 2^{k}}\left|\hat{H}_{\tau, n^{\prime}}\right|^{2}\right. \\
& \left.\left.\times\left|\hat{F}_{\tau-\tau_{1}, n^{\prime}-n_{1}^{\prime}}\right|^{2} d \tau d \tau_{1}\right]^{1 / 2}\right\} \\
& \leq C N \sum_{n_{1} \in \mathbb{N}} \sup _{k_{1} \in \mathbb{N}}\left(\sum_{\left|n_{1}^{\prime}\right| \sim 2^{n_{1}}} \int_{\left|\tau_{1}\right| \sim 2^{k_{1}}}\left|\hat{G}_{\tau_{1}, n_{1}^{\prime}}\right|^{2} d \tau_{1}\right)^{1 / 2} \\
& \times \sup _{\substack{n_{1} \in \mathbb{N} \\
k_{1} \in \mathbb{N}}} \sup _{\substack{n_{0} \leq n \leq n_{0}+N \\
k_{0} \leq k \leq k_{0}+N}}\left(\sum_{\left|n^{\prime}\right| \sim 2^{n}} \int_{|\tau| \sim 2^{k}}\left|\hat{H}_{\tau, n^{\prime}}\right|^{2} d \tau\right)^{1 / 2} \\
& \times \sup _{n_{1} \in \mathbb{N}} \sum_{k_{1} \in \mathbb{N}} \sup _{\substack{n_{0} \leq n \leq n_{0}+N \\
k_{0} \leq k \leq k_{0}+N}} \sup _{\substack{\left|n^{\prime}\right| \sim 2^{n} \\
|\tau| \sim 2^{k}}}\left(\sum_{\substack{\left|n_{1}^{\prime}\right| \sim 2^{n_{1}} \\
k^{\prime}}} \int_{\left|\tau_{1}\right| \sim 2^{k_{1}}}\left|\hat{F}_{\tau-\tau_{1}, n^{\prime}-n_{1}^{\prime}}\right|^{2} d \tau_{1}\right)^{1 / 2} \\
& \leq C N|G|_{X_{1, \infty}^{0,0}}|H|_{X_{\infty, \infty}^{0,0}} \\
& \times \sup _{n_{1} \in \mathbb{N}} \sum_{k_{1} \in \mathbb{N}} \sup _{\substack{n_{0} \leq n \leq n_{0}+N \\
k_{0} \leq k \leq k_{0}+N}} \sup _{\substack{\left|n^{\prime}\right| \sim 2^{n} \\
|\tau| \sim 2^{k}}}\left(\sum_{\substack{\left|n_{1}^{\prime}\right| \sim 2^{n_{1}}\\
}} \int_{\left|\tau_{1}\right| \sim 2^{k_{1}}}\left|\hat{F}_{\tau-\tau_{1}, n^{\prime}-n_{1}^{\prime}}\right|^{2} d \tau_{1}\right)^{1 / 2} .
\end{aligned}
$$

It remains to bound the last term in the right hand side above by $C|F|_{X_{1,1}^{0,0}}$. However, this is not completely obvious, and we again have to consider separately each of the regions I-c-1, I-c-2 and I-c-3.

Region I-c-2. Recall that we have here : $\left|n^{\prime}\right| \leq 2^{-12}\left|n_{1}^{\prime}\right|$. 
Then the last term in the right hand side of the above inequality is clearly bounded by

$$
2 \sup _{n_{1} \in \mathbb{N}} \sum_{k_{1} \in \mathbb{N}} \sum_{\substack{n=n_{0}\left(n_{1}, k_{1}\right) \\ n \leq n_{1}-10}}^{n_{0}+N} \sum_{k=k_{0}}^{k_{0}+N}\left(\sum_{\left|n_{1}^{\prime}\right| \sim 2^{n_{1}}} \int_{\left|\tau_{1}\right| \sim 2^{k_{1}}}\left|\hat{F}_{\tau-\tau_{1}, n^{\prime}-n_{1}^{\prime}}\right|^{2} d \tau_{1}\right)^{1 / 2} .
$$

- The contribution to this term of the $k$ and $k_{1}$ for which $k \leq k_{1}-4$ or $k \geq k_{1}+4$ is clearly bounded above by

$$
\begin{aligned}
& 2 N^{2} \sup _{n_{1} \in \mathbb{N}} \sum_{k \in \mathbb{N}}\left(\sum_{\left|n_{1}^{\prime}\right| \sim 2^{n_{1}}} \int_{|\tau| \sim 2^{k}}\left|\hat{F}_{\tau, n_{1}^{\prime}}\right|^{2} d \tau\right)^{1 / 2} \\
& \leq 2 N^{2}|F|_{X_{1,1}^{0,0}}
\end{aligned}
$$

- It remains to consider in the sum in $k_{1}$ and $k$, the contribution of the terms for which $k_{1}-4 \leq k \leq k_{1}+4$. Since for such terms, $\tau-\tau_{1}$ may stay bounded, we need to show that there are only a finite number of possibilities for $k_{1}$. We recall that in Region I-c, $k \leq 3 n+4$, while in Region I-c-2, $n \leq n_{1}-10$; it follows easily that if in addition $k_{1}-4 \leq k \leq k_{1}+4$, then $k_{1} \leq 3 n_{1}-4$. Hence, $n_{0}\left(n_{1}, k_{1}\right)=\ln \frac{\left|2^{k_{1}}-2^{3 n_{1}}\right|}{\ln 2}-2 n_{1}=n_{1}-1$ and the region is actually empty.

Region I-c-3: $\left|n^{\prime}\right| \geq 2^{12}\left|n_{1}^{\prime}\right|$.

Again, the last term in (3.7) is easily bounded above by

$$
2 \sup _{n_{1} \in \mathbb{N}} \sum_{k_{1} \in \mathbb{N}} \sum_{\substack{n=n_{0}\left(n_{1}, k_{1}\right) \\ n \geq n_{1}+3}}^{n_{0}+N} \sum_{k=k_{0}}^{k_{0}+N} \sup _{|\tau| \sim 2^{k}}\left(\sum_{\left|n^{\prime}\right| \sim 2^{n}} \int_{\left|\tau_{1}\right| \sim 2^{k_{1}}}\left|\hat{F}_{\tau-\tau_{1}, n^{\prime}}\right|^{2} d \tau_{1}\right)^{1 / 2}
$$

- in the same way as before, the contribution in this sum of the terms for which $k \leq k_{1}-4$ or $k \geq k_{1}+4$ is bounded by

$$
2 N^{2} \sup _{n \in \mathbb{N}} \sum_{k \in \mathbb{N}}\left(\sum_{\left|n^{\prime}\right| \sim 2^{n}} \int_{|\tau| \sim 2^{k}}\left|\hat{F}_{\tau, n^{\prime}}\right|^{2} d \tau\right)^{1 / 2} \leq 2 N^{2}|F|_{X_{1,1}^{0,0}}
$$

- in the region where $k_{1}-4 \leq k \leq k_{1}+4$, we easily get $k_{1} \geq 3 n_{1}+4$, and from the expression of $n_{0}\left(n_{1}, k_{1}\right)$ in region I-c-3, we get $n_{0}\left(n_{1}, k_{1}\right)=\frac{1}{2}\left(k_{1}-n_{1}\right)$. Hence, $n \geq \frac{1}{2}(3 n-8)-\frac{1}{2} n_{1}$ from which it follows that $n_{1} \geq n-8$, and again the region is empty, since $n \geq n_{1}+10$.

Region I-c-1 : $2^{-12}\left|n_{1}^{\prime}\right| \leq\left|n^{\prime}\right| \leq 2^{12}\left|n_{1}^{\prime}\right|$. 
This is the most difficult part; clearly, we can take in this region $n_{0}\left(n_{1}, k_{1}\right)=$ $n_{1}$. Again, we will divide the region into three subregions depending on the size of $k$ and $k_{1}$ compared to each other.

- $k \leq k_{1}-4$ : the contribution of this region to the last term in (3.7) is then bounded above by

$$
2 \sup _{n_{1} \in \mathbb{N}} \sum_{k_{1} \in \mathbb{N}} \sum_{n=n_{1}}^{n_{1}+N} \sup _{\left|n^{\prime}\right| \sim 2^{n}}\left(\sum_{\left|n_{1}^{\prime}\right| \sim 2^{n_{1}}} \int_{\left|\tau_{1}\right| \sim 2^{k_{1}}}\left|\hat{F}_{\tau_{1}, n^{\prime}-n_{1}^{\prime}}\right|^{2} d \tau_{1}\right)^{1 / 2} .
$$

Now, since for each $n_{1}, k_{1}, n$ and $n^{\prime}$ such that $\left|n^{\prime}\right| \sim 2^{n}$, one has

$$
\begin{aligned}
& \left(\sum_{\left|n_{1}^{\prime}\right| \sim 2^{n_{1}}} \int_{\left|\tau_{1}\right| \sim 2^{k_{1}}}\left|\hat{F}_{\tau_{1}, n^{\prime}-n_{1}^{\prime}}\right|^{2} d \tau_{1}\right)^{1 / 2} \\
& \leq \sum_{\ell \in \mathbb{N}}\left(\sum_{\left|\ell^{\prime}\right| \sim 2^{\ell}} \int_{\left|\tau_{1}\right| \sim 2^{k_{1}}}\left|\hat{F}_{\tau_{1}, \ell^{\prime}}\right|^{2} d \tau_{1}\right)^{1 / 2},
\end{aligned}
$$

the preceding term is easily bounded above by

$$
\begin{aligned}
& 2 \sup _{n_{1} \in \mathbb{N}} \sum_{n=n_{1}}^{n_{1}+N} \sum_{k_{1} \in \mathbb{N}} \sum_{\ell \in \mathbb{N}}\left(\sum_{\left|\ell^{\prime}\right| \sim 2^{\ell}} \int_{\left|\tau_{1}\right| \sim 2^{k_{1}}}\left|\hat{F}_{\tau_{1}, \ell^{\prime}}\right|^{2} d \tau_{1}\right)^{1 / 2} \\
& \leq 2 N|F|_{X_{1,1}^{0,0}}
\end{aligned}
$$

- $k_{1}-4 \leq k \leq k_{1}+4$ : Using again the arguments immediately above the contribution of this region to the last term in (3.7) may be bounded above by

$$
\begin{aligned}
& \sup _{n_{1} \in \mathbb{N}} \sum_{k_{1} \in \mathbb{N}} \sup _{\substack{n_{1} \leq n \leq n_{1}+N \\
3 n \leq k \leq 3 n+N \\
k_{1}-4 \leq k \leq k_{1}+4}} \sup _{|\tau| \sim 2^{k}} \sum_{\ell \in \mathbb{N}}\left(\sum_{\left|\ell^{\prime}\right| \sim 2^{\ell}} \int_{\left|\tau_{1}\right| \sim 2^{k_{1}}}\left|\hat{F}_{\tau-\tau_{1}, \ell^{\prime}}\right|^{2} d \tau_{1}\right)^{1 / 2} \\
& \leq \sup _{n_{1} \in \mathbb{N}} \sum_{k_{1} \in \mathbb{N}} \sup _{\substack{3 n_{1} \leq k \leq 3 n_{1}+4 N \\
k_{1}-4 \leq k \leq k_{1}+4}} \sup _{|\tau| \sim 2^{k}} \sum_{\ell \in \mathbb{N}}\left(\sum_{\left|\ell^{\prime}\right| \sim 2^{\ell}} \int_{\left|\tau_{1}\right| \sim 2^{k_{1}}}\left|\hat{F}_{\tau-\tau_{1}, \ell^{\prime}}\right|^{2} d \tau_{1}\right)^{1 / 2} .
\end{aligned}
$$

Here again, $\tau-\tau_{1}$ may stay bounded even for large $k$ and $k_{1}$; however, for a fixed $n_{1}$, the number of $k_{1}$ for which the right hand side gives a nonzero contribution is bounded by the total number of $k_{1}$ for which there exists at least one $k$ such that $3 n_{1} \leq k \leq 3 n_{1}+4 N$ and $k_{1}-4 \leq k \leq k_{1}+4$, hence by 
$4 N+8$. In this way, the term above is bounded by

$$
\begin{aligned}
& (4 N+8) \sup _{n_{1} \in \mathbb{N}} \sup _{k, k_{1} \in \mathbb{N}} \sup _{|\tau| \sim 2^{k}} \sum_{\ell \in \mathbb{N}}\left(\sum_{\left|\ell^{\prime}\right| \sim 2^{\ell}} \int_{\left|\tau_{1}\right| \sim 2^{k_{1}}}\left|\hat{F}_{\tau-\tau_{1}, \ell^{\prime}}\right|^{2} d \tau_{1}\right)^{1 / 2} \\
& \leq(4 N+8) \sum_{\ell \in \mathbb{N}} \sum_{j \in \mathbb{N}}\left(\sum_{\left|\ell^{\prime}\right| \sim 2^{\ell}} \int_{|\tau| \sim 2^{j}}\left|\hat{F}_{\tau, \ell^{\prime}}\right|^{2} d \tau\right)^{1 / 2} \\
& \leq(4 N+8)|F|_{X_{1,1}^{0,0}}
\end{aligned}
$$

- $k \geq k_{1}+4$ : this region is a little bit more delicate than the preceding ones to handle. In the same way as before, we may bound above the contribution of the present region to the last term in the right hand side of (3.7) by

$$
\begin{aligned}
& \sup _{n_{1} \in \mathbb{N}} \sum_{k_{1} \in \mathbb{N}} \sup _{\substack{3 n_{1} \leq k \leq 3 n_{1}+2 N \\
k \geq k_{1}+4}} \sup _{\substack{|\tau| \sim 2^{k} \\
\ell \in \mathbb{N}}} \sum_{\ell}\left(\sum_{\left|\ell^{\prime}\right| \sim 2^{\ell}} \int_{\left|\tau_{1}\right| \sim 2^{k_{1}}}\left|\hat{F}_{\tau-\tau_{1}, \ell^{\prime}}\right|^{2} d \tau_{1}\right)^{1 / 2} \\
& \leq 2 \sup _{n_{1} \in \mathbb{N}} \sum_{k_{1} \in \mathbb{N}} \sup _{\substack{3 n_{1} \leq k \leq 3 n_{1}+2 N \\
k \geq k_{1}+4}} \sum_{\ell \in \mathbb{N}}\left(\sum_{\left|\ell^{\prime}\right| \sim 2^{\ell}} \int_{|\tau| \sim 2^{k}}\left|\hat{F}_{\tau, \ell^{\prime}}\right|^{2} d \tau\right)^{1 / 2} .
\end{aligned}
$$

Again, we have to show that the number of possible $k_{1}$ (or $n$ or $n_{1}$ ) in this region is finite. We recall that here, $n$ and $n^{\prime}$ are of the same order ; moreover, since $\left|\tau-n^{\prime 3}\right| \leq \frac{1}{4}\left|n^{\prime}\right|^{2}$ and $\left|\tau-\tau_{1}-\left(n^{\prime}-n_{1}^{\prime}\right)^{3}\right| \leq \frac{1}{4}\left|n^{\prime}\right|^{2}$, it follows that $|\tau|$ is of the order of $\left|n^{\prime}\right|^{3}$; then $\left|\tau_{1}\right|$, which is negligible compared with $|\tau|$, is negligible compared with $\left|n^{\prime}\right|^{3}$. Hence $\tau_{1}-n_{1}^{\prime 3} \sim-n_{1}^{\prime 3}$ for $n_{1}$ sufficiently large. Now, we have the relation

$$
\tau_{1}-n_{1}^{\prime 3}-\tau+n^{\prime 3}+\tau-\tau_{1}-\left(n^{\prime}-n_{1}^{\prime}\right)^{3}=3 n^{\prime} n_{1}^{\prime}\left(n^{\prime}-n_{1}^{\prime}\right) .
$$

- Consider first the case where $n^{\prime}$ and $n_{1}^{\prime}$ have opposite signs. Then, taking into account the preceding considerations, one may note that the left hand side in (3.9) is of the order of $-n_{1}^{\prime 3}$ (for $\left|n_{1}^{\prime}\right|$ large) while the right hand side has the sign of $n_{1}^{\prime 3}$. Hence (3.9) cannot remain true for large $\left|n_{1}^{\prime}\right|$, which implies that the number of $n_{1}^{\prime}$ in this region is finite.

- Now, if $n^{\prime}$ and $n_{1}^{\prime}$ have the same sign, then comparing the sign of both sides in (3.9) shows that if $\left|n_{1}^{\prime}\right|$ is large, then necessarily, $n^{\prime}-n_{1}^{\prime}$ has a sign opposite to that of $n_{1}^{\prime}$. But then, for $\left|n^{\prime}\right|$ (or equivalently for $\left|n_{1}^{\prime}\right|$ ) large, the facts that $\tau \sim n^{\prime 3}, \tau-\tau_{1} \sim\left(n^{\prime}-n_{1}^{\prime}\right)^{3}$ and $\tau_{1}$ is negligible compared with $\tau$ lead again to incompatible signs. 
This shows that, in any case, the number of possible $n_{1}$ (or $n$, or $k_{1}$ ) in this region is finite. Hence, (3.8) is bounded above by

$$
\begin{aligned}
& C \sup _{n_{1}} \sup _{k_{1}} \sup _{3 n_{1} \leq k \leq 3 n_{1}+2 N} \sum_{\ell}\left(\sum_{\left|\ell^{\prime}\right| \sim 2^{\ell}} \int_{|\tau| \sim 2^{k}}\left|\hat{F}_{\tau, \ell^{\prime}}\right|^{2} d \tau\right)^{1 / 2} \\
& \leq C|F|_{X_{1,1}^{0,0} .}
\end{aligned}
$$

This ends the proof of the required estimate in Region I, that is when $\left\langle\sigma_{1}\right\rangle$ dominates.

Region II. Here, we use the fact that

$$
\frac{1}{2}\left|n^{\prime}\right|^{2} \leq\left|n^{\prime} n_{1}^{\prime}\left(n^{\prime}-n_{1}^{\prime}\right)\right| \leq\langle\sigma\rangle
$$

so that for any $s \in\left[-\frac{1}{2}, 0\right]$,

$$
\left|n^{\prime}\right|^{1+s}\left|n_{1}^{\prime}\right|^{-s}\left|n^{\prime}-n_{1}^{\prime}\right|^{-s} \leq C\langle\sigma\rangle^{1 / 2} .
$$

Exchanging then the role of $n^{\prime}$ and $n_{1}^{\prime}$ - and hence of $\hat{G}$ and $\hat{H}$ - we are led back to prove that the contribution of Region $I$ to $I$ is bounded above by $C|H|_{X_{1, \infty}^{0,0}}|G|_{X_{\infty, \infty}^{0,0}}|F|_{X_{1,1}^{0,0}}$

For Regions I-a and I-b, this was already done, since the contribution of Regions I-a and I-b to $I$ was actually bounded above by $C|H|_{X_{\infty, \infty}^{0,0}}|G|_{X_{\infty, \infty}^{0,0}}|F|_{X_{1,1}^{0,0} \text {. }}$

It remains only to consider the case of Region I-c. Again, the same computations as before lead to bound the contribution of Region I-c to $I$ as in (3.7), except that the sum over $n_{1}$ will be supported by $\hat{H}$ or $\hat{F}$, so that this contribution is bounded by (see (3.7))

$$
\begin{aligned}
& C N \sup _{n_{1} \in \mathbb{N}} \sup _{k_{1} \in \mathbb{N}}\left(\sum_{\left|n_{1}^{\prime}\right| \sim 2^{n_{1}}} \int_{\left|\tau_{1}\right| \sim 2^{k_{1}}}\left|\hat{G}_{\tau_{1}, n_{1}^{\prime}}\right|^{2} d \tau_{1}\right)^{1 / 2} \\
& \quad \times \sum_{n_{1} \in \mathbb{N}}\left\{\sup _{k_{1} \in \mathbb{N}} \sup _{n_{0} \leq n \leq n_{0}+N} \sup _{k_{0} \leq k \leq k_{0}+N}\left(\sum_{\left|n^{\prime}\right| \sim 2^{n}} \int_{|\tau| \sim 2^{k}}\left|\hat{H}_{\tau, n^{\prime}}\right|^{2} d \tau\right)^{1 / 2}\right. \\
& \left.\quad \times \sum_{k_{1} \in \mathbb{N}} \sup _{n_{0} \leq n \leq n_{0}+n} \sup _{k_{0} \leq k \leq k_{0}+N} \sup _{\substack{\left|n^{\prime}\right| \sim 2^{n} \\
|\tau| \sim 2^{k}}}\left(\sum_{\left|n_{1}^{\prime}\right| \sim 2^{n_{1}}} \int_{\left|\tau_{1}\right| \sim 2^{k_{1}}}\left|\hat{F}_{\tau-\tau_{1}, n^{\prime}-n_{1}^{\prime}}\right|^{2} d \tau_{1}\right)^{1 / 2}\right\} .
\end{aligned}
$$

Hence, we have to bound above the two last lines in (3.10) by $C|H|_{X_{1, \infty}^{0,0}}|F|_{X_{1,1}^{0,0}}$.

Considering the way we have estimated the contribution of Regions I-c-2 and I-c-3 to (3.7), it is clear that the sum over $k_{1}$ in these regions can be 
supported by $\left|\hat{F}_{\tau-\tau_{1}, n^{\prime}-n_{1}^{\prime}}\right|^{2}$; in Region I-c-1, we have $2^{-12}\left|n_{1}^{\prime}\right| \leq\left|n^{\prime}\right| \leq 2^{12}\left|n_{1}^{\prime}\right|$ so that $n_{0}\left(n_{1}, k_{1}\right)=n_{1}$ and

$$
\begin{aligned}
& \sum_{n_{1} \in \mathbb{N}} \sup _{k_{1} \in \mathbb{N}} \sup _{n_{0} \leq n \leq n_{0}+N} \sup _{k_{0} \leq k \leq k_{0}+N}\left(\sum_{\left|n^{\prime}\right| \sim 2^{n}} \int_{|\tau| \sim 2^{k}}\left|\hat{H}_{\tau, n^{\prime}}\right|^{2} d \tau\right)^{1 / 2} \\
& \leq C \sum_{n_{1} \in \mathbb{N}} \sup _{k_{1} \in \mathbb{N}}\left(\sum_{\left|n^{\prime}\right| \sim 2^{n_{1}}} \int_{\left|\tau_{1}\right| \sim 2^{k_{1}}}\left|\hat{H}_{\tau_{1}, n_{1}^{\prime}}\right|^{2} d \tau_{1}\right)^{1 / 2} \\
& \leq C|H|_{X_{1, \infty}^{0,0}} .
\end{aligned}
$$

We may conclude as before.

Region III. Again, exchanging the role of $\hat{G}$ and $\hat{F}$, we are led back to prove that the contribution of Region I to I is bounded above by $C|G|_{X_{1,1}^{0,0}}|H|_{X_{\infty, \infty}^{0,0}}|F|_{X_{1, \infty}^{0,0}}$, but this is easily done by using the same analysis as for Region I. Hence, the proof of Proposition 3.1 is complete.

We now prove that when local in time spaces are considered, that is when $X_{1,1}^{s,-1 / 2}$ is replaced by $X_{1,1}^{s,-1 / 2, T}$, a small power of $T$ can be recovered in the right hand side of the estimate in Proposition 3.1. This will be useful in the contraction procedure, since as is now classical, no small power of $T$ is gained, but on the opposite a $\ln T$ factor is lost in the estimate of the integral convolution with the linear semi-group when dealing with spaces of regularity $1 / 2$ in time.

The argument of the proof of the next proposition relies, as usual, on the fact that we have wasted a small power of $\langle\sigma\rangle$ or $\left\langle\sigma_{2}\right\rangle$ in Lemma 3.1. Actually, looking carefully to the proof shows that Lemma 3.1 is still true with $B\left(n_{1}, k_{1}, n, k\right)$ replaced by

$$
\tilde{B}\left(n_{1}, k_{1}, n, k\right)=\sup _{\substack{\left|n_{1}^{\prime}\right| \sim 2^{n_{1}} \\\left|\tau_{1}\right| \sim 2^{k_{1}}}}\left(\sum_{\left|n^{\prime}\right| \sim 2^{n}} \int_{|\tau| \sim 2^{k}} \frac{d \tau}{\langle\sigma\rangle^{1-\varepsilon}\left\langle\sigma_{2}\right\rangle^{1-\varepsilon}}\right)^{1 / 2}
$$

for any $\varepsilon<1 / 4$.

Proposition 3.2. Let $-1 / 2 \leq s \leq 0$ and $f \in X_{1,1}^{s, 1 / 2, T}, g \in X_{1, \infty}^{s, 1 / 2, T}$; then for any $\alpha<1 / 16$, there is a constant $C_{\alpha}$ such that

$$
\left|\partial_{x}(f g)\right|_{X_{1,1}^{s,-1 / 2, T}} \leq C_{\alpha} T^{\alpha}|f|_{X_{1,1}^{s, 1 / 2, T}}|g|_{X_{1, \infty}^{s, 1 / 2, T}}
$$


Proof. Let $f \in X_{1,1}^{s, 1 / 2}, g \in X_{1, \infty}^{s, 1 / 2}, s \geq 1 / 2$, both with support in $[-2 T, 2 T]$. Using the arguments immediately above shows that we have actually proved, during the course of the proof of Proposition 3.1, that

$$
\left|\partial_{x}(f g)\right|_{X_{1,1}^{s,-1 / 2}} \leq C\left(|f|_{X_{1,1}^{s, 1 / 2}}|g|_{X_{1, \infty}^{s, \delta}}+|f|_{X_{1,1}^{s, \delta}}|g|_{X_{1, \infty}^{s, 1 / 2}}\right)
$$

for any $\delta>3 / 8$. Let $s=0$ (the arguments are exactly the same in the other cases) and $\delta$ such that $3 / 8<\delta<1 / 2$. By an obvious interpolation inequality, one gets

$$
|g|_{X_{1, \infty}^{0, \delta}} \leq C|g|_{X_{1, \infty}^{0,0}}^{1-2 \delta}|g|_{X_{1, \infty}^{0,1 / 2}}^{2 \delta}
$$

On the other hand, using the notations introduced at the beginning of Section 2, we have

$$
\begin{aligned}
|g|_{X_{1, \infty}^{0,0}} & =\sum_{n=0}^{\infty} \sup _{k \in \mathbb{N}}\left(\sum_{n^{\prime} \in \mathbb{N} \backslash\{0\}} \int_{|\tau| \sim 2^{k}}\left|\widehat{\Delta_{n} g}\left(\tau, n^{\prime}\right)\right|^{2} d \tau\right)^{1 / 2} \\
& \leq \sum_{n=0}^{\infty}\left|\Delta_{n} g\right|_{L_{x, t}^{2}([-2 T, 2 T] \times \mathbb{T})} \\
& \leq C T^{1 / 4} \sum_{n=0}^{\infty}\left|\Delta_{n} g\right|_{L_{x, t}^{4}([-2 T, 2 T] \times \mathbb{T})} \\
& \leq C T^{1 / 4} \sum_{n=0}^{\infty}\left(\sum_{n^{\prime} \in \mathbb{N} \backslash\{0\}} \int_{\tau \in \mathbb{R}}\langle\sigma\rangle^{2 / 3}\left|\widehat{\Delta_{n} g}\left(\tau, n^{\prime}\right)\right|^{2} d \tau\right)^{1 / 2}
\end{aligned}
$$

where we have used in the last line above the Strichartz estimate proved in [4]. It follows readily that

$$
|g|_{X_{1, \infty}^{0,0}} \leq C T^{1 / 4}|g|_{X_{1,2}^{0,1 / 3}} \leq C T^{1 / 4}|g|_{X_{1, \infty}^{0,1 / 2}}
$$

and from the above interpolation inequality,

$$
|g|_{X_{1, \infty}^{0, \delta}} \leq C T^{(1-2 \delta) / 4}|g|_{X_{1, \infty}^{0,1 / 2}}
$$

In the same way, we estimate $f$ as follows : taking a small positive $\varepsilon$, one has

$$
|f|_{X_{1,1}^{0, \delta}} \leq C|f|_{X_{1,1}^{0,-\varepsilon}}^{(1-2 \delta) /(1+2 \varepsilon)}|f|_{X_{1,1}^{0,1 / 2}}^{2(\varepsilon+\delta) /(1+2 \varepsilon)}
$$

and

$$
|f|_{X_{1,1}^{0,-\varepsilon}} \leq C|f|_{X_{1,2}^{0,0}} \leq C T^{1 / 4}|f|_{X_{1,2}^{0,1 / 3}}
$$

by using again the estimate in [4] for $\Delta_{n} f$; it follows that

$$
|f|_{X_{1,1}^{0,-\varepsilon}} \leq C T^{1 / 4}|f|_{X_{1,1}^{0,1 / 2}}
$$

Finally,

$$
\left|\partial_{x}(f g)\right|_{X_{1,1}^{0,-1 / 2}} \leq C_{\alpha} T^{\alpha}|f|_{X_{1,1}^{0,1 / 2}}|g|_{X_{1, \infty}^{0,1 / 2}}
$$


where $\alpha$ is chosen such that $\alpha<(1-2 \delta) / 4$, with $\delta>3 / 8$, so that at the very end, $\alpha<1 / 16$, and since $f$ and $g$ have supports in $[-2 T, 2 T]$, the proof of Proposition 3.2 follows.

We now prove an estimate of the same type as those in Propositions 3.1 and 3.2, but in $Y_{s}$ spaces. We recall that the use of these spaces is needed to handle the integral estimate in Duhamel's formula (see Proposition 4.1).

Proposition 3.3. Let $-1 / 2 \leq s \leq 0, f \in X_{1,1}^{s, 1 / 2}, g \in X_{1, \infty}^{s, 1 / 2}$; then $\partial_{x}(f g) \in$ $Y_{s}$. Moreover, for any $\alpha<1 / 16$, there is a constant $C_{\alpha}>0$ such that

$$
\left|\partial_{x}(f g)\right|_{Y_{s, T}} \leq C_{\alpha} T^{\alpha}|f|_{X_{1,1}^{s, 1 / 2, T}}|g|_{X_{1, \infty}^{s, 1 / 2, T}}
$$

Proof. We only sketch the proof, since it is a slight modification of the proof of Proposition 3.1, using e.g. the arguments in [20]. Let $f \in X_{1,1}^{s, 1 / 2}$ and $g \in X_{1, \infty}^{s, 1 / 2}$. We only prove the estimate

$$
\left|\partial_{x}(f g)\right|_{Y_{s}} \leq C|f|_{X_{1,1}^{s, 1 / 2}}|g|_{X_{1, \infty}^{s, 1 / 2}}
$$

the $T^{\alpha}$ factor can indeed be recovered exactly as in the proof of Proposition 3.2 .

By a duality argument, the estimate will be proved if we show that there is a constant $C>0$ such that for any function $w$ (of the space variable $x$ ) lying in the Besov space $B_{2, \infty}^{-s}$, one has

$$
\left|\sum_{n^{\prime} \neq 0}\right| n^{\prime}\left|\int_{\mathbb{R}} \frac{\widehat{f g}\left(\tau, n^{\prime}\right)}{\left\langle\sigma\left(\tau, n^{\prime}\right)\right\rangle} d \tau \hat{w}\left(n^{\prime}\right)\right| \leq C|f|_{X_{1,1}^{s, 1 / 2}}|g|_{X_{1, \infty}^{s, 1 / 2}}|w|_{B_{2, \infty}^{-s}} .
$$

Setting as above $\hat{F}\left(\tau, n^{\prime}\right)=n^{\prime s}\left\langle\sigma\left(\tau, n^{\prime}\right)\right\rangle^{1 / 2} \hat{f}\left(\tau, n^{\prime}\right), \hat{G}=n^{\prime s}\langle\sigma\rangle^{1 / 2} \hat{g}$ and $\hat{W}=$ $n^{\prime-s} \hat{w}$, it suffices to prove that

$$
\begin{aligned}
& \sum_{n^{\prime} \neq 0} \sum_{\substack{n_{1}^{\prime} \neq 0 \\
n_{1}^{\prime} \neq n^{\prime}}} \int_{\tau \in \mathbb{R}} \int_{\tau_{1} \in \mathbb{R}} \frac{\left|n^{\prime}\right|^{1+s}\left|n_{1}^{\prime}\right|^{-s}\left|n^{\prime}-n_{1}^{\prime}\right|^{-s}\left|\hat{F}_{\tau-\tau_{1}, n^{\prime}-n_{1}^{\prime}}\right|\left|\hat{G}_{\tau_{1}, n_{1}^{\prime}}\right|\left|\hat{W}_{n^{\prime}}\right|}{\left\langle\sigma\left(\tau, n^{\prime}\right)\right\rangle\left\langle\sigma\left(\tau-\tau_{1}, n^{\prime}-n_{1}^{\prime}\right)\right\rangle^{1 / 2}\left\langle\sigma\left(\tau_{1}, n_{1}^{\prime}\right)\right\rangle^{1 / 2}} d \tau d \tau_{1} \\
& \leq C|F|_{X_{1,1}^{0,0}|G|_{X_{1, \infty}^{0,0}}|W|_{B_{2, \infty}^{0}} .}
\end{aligned}
$$

Again, we will consider separately the three regions defined at the beginning of the proof of Proposition 3.1.

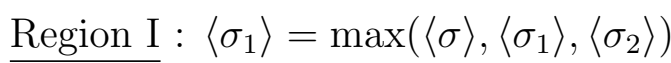


As already noticed, we have in this region

$$
\left|n^{\prime}\right|^{1+s}\left|n_{1}^{\prime}\right|^{-s}\left|n^{\prime}-n_{1}^{\prime}\right|^{-s} \leq C\left\langle\sigma\left(\tau_{1}, n_{1}^{\prime}\right)\right\rangle^{1 / 2} .
$$

Hence, taking $\varepsilon>0$ small, we have

$$
\begin{aligned}
& \frac{\left|n^{\prime}\right|^{1+s}\left|n_{1}^{\prime}\right|^{-s}\left|n^{\prime}-n_{1}^{\prime}\right|^{-s}\left|\hat{F}_{\tau-\tau_{1}, n^{\prime}-n_{1}^{\prime}}\right|\left|\hat{G}_{\tau_{1}, n_{1}^{\prime}}\right|\left|\hat{W}_{n^{\prime}}\right|}{\left\langle\sigma\left(\tau, n^{\prime}\right)\right\rangle\left\langle\sigma\left(\tau-\tau_{1}, n^{\prime}-n_{1}^{\prime}\right)\right\rangle^{1 / 2}\left\langle\sigma\left(\tau_{1}, n_{1}^{\prime}\right)\right\rangle^{1 / 2}} \\
& \leq C \frac{\left|\hat{W}_{n^{\prime}}\right|}{\left\langle\sigma\left(\tau, n^{\prime}\right)\right\rangle^{1 / 2+\varepsilon}} \frac{\left|\hat{F}_{\tau-\tau_{1}, n^{\prime}-n_{1}^{\prime}}\right|\left|\hat{G}_{\tau_{1}, n_{1}^{\prime}}\right|}{\left\langle\sigma\left(\tau, n^{\prime}\right)\right\rangle^{1 / 2-\varepsilon}\left\langle\sigma\left(\tau-\tau_{1}, n^{\prime}-n_{1}^{\prime}\right)\right\rangle^{1 / 2}}
\end{aligned}
$$

and we conclude as in the proof of Proposition 3.1, using the fact that $\mathcal{F}^{-1}\left(\frac{\hat{W}}{\langle\sigma\rangle^{1 / 2+\varepsilon}}\right) \in X_{\infty, \infty}^{0,0}$, with

$$
\left|\mathcal{F}^{-1}\left(\frac{\hat{W}}{\langle\sigma\rangle^{1 / 2+\varepsilon}}\right)\right|_{X_{\infty, \infty}^{0,0}} \leq C_{\varepsilon}|W|_{B_{2, \infty}^{0}}
$$

and using again the fact that Lemma 3.1 is still true with a little smaller power of $\sigma\left(\tau, n^{\prime}\right)$.

Region III, that is $\left\langle\sigma_{2}\right\rangle=\max \left(\langle\sigma\rangle,\left\langle\sigma_{1}\right\rangle,\left\langle\sigma_{2}\right\rangle\right)$ is treated in the same way.

Region II : $\langle\sigma\rangle=\max \left(\langle\sigma\rangle,\left\langle\sigma_{1}\right\rangle,\left\langle\sigma_{2}\right\rangle\right)$.

Here, we have

$$
\left|n^{\prime}\right|^{1+s}\left|n_{1}^{\prime}\right|^{-s}\left|n^{\prime}-n_{1}^{\prime}\right|^{-s} \leq C\left\langle\sigma\left(\tau, n^{\prime}\right)\right\rangle^{1 / 2}
$$

and it follows that

$$
\frac{1}{\left\langle\sigma\left(\tau, n^{\prime}\right)\right\rangle} \leq \frac{C}{\left\langle\sigma\left(\tau, n^{\prime}\right)\right\rangle+\left|n^{\prime}\right|^{2+2 s}\left|n_{1}^{\prime}\right|^{-2 s}\left|n^{\prime}-n_{1}^{\prime}\right|^{-2 s}} .
$$

Hence, going back to the way we have proved Proposition 3.1, it suffices to show that for a fixed $n_{1}^{\prime}$,

$$
\mathcal{F}^{-1}\left(\frac{\left|n^{\prime}\right|^{1+s}\left|n_{1}^{\prime}\right|^{-s}\left|n^{\prime}-n_{1}^{\prime}\right|^{-s} \hat{W}\left(n^{\prime}\right)}{\left\langle\sigma\left(\tau, n^{\prime}\right)\right\rangle+\left|n^{\prime}\right|^{2+2 s}\left|n_{1}^{\prime}\right|^{-2 s}\left|n^{\prime}-n_{1}^{\prime}\right|^{-2 s}}\right) \in X_{\infty, \infty}^{0,0}
$$

with

$$
\left|\mathcal{F}^{-1}\left(\frac{\left|n^{\prime}\right|{ }^{1+s}\left|n_{1}^{\prime}\right|^{-s}\left|n^{\prime}-n_{1}^{\prime}\right|^{-s} \hat{W}\left(n^{\prime}\right)}{\left\langle\sigma\left(\tau, n^{\prime}\right)\right\rangle+\left|n^{\prime}\right|^{2+2 s}\left|n_{1}^{\prime}\right|^{-2 s}\left|n^{\prime}-n_{1}^{\prime}\right|^{-2 s}}\right)\right|_{X_{\infty, \infty}^{0,0}} \leq C|W|_{B_{2, \infty}^{0}}
$$

and a constant $C$ that does not depend on $n_{1}^{\prime}$. 
But this follows from the next easy computation, once we have noticed that

$$
\begin{aligned}
\int_{\mathbb{R}} \frac{d \tau}{\left(\langle\tau\rangle+a^{2}\right)^{2}} \leq \frac{C}{a^{2}}: \\
\quad\left|\mathcal{F}^{-1}\left(\frac{\left|n^{\prime}\right|^{1+s}\left|n_{1}^{\prime}\right|^{-s}\left|n^{\prime}-n_{1}^{\prime}\right|^{-s} \hat{W}\left(n^{\prime}\right)}{\left\langle\sigma\left(\tau, n^{\prime}\right)\right\rangle+\left|n^{\prime}\right|^{2+2 s}\left|n_{1}^{\prime}\right|^{-2 s}\left|n^{\prime}-n_{1}^{\prime}\right|^{-2 s}}\right)\right|_{X_{\infty}^{0,0}, \infty}^{2} \\
=\sup _{n, k} \sum_{\left|n^{\prime}\right| 2^{n}} \int_{|\tau| \sim 2^{k}} \frac{\left|n^{\prime}\right|^{2+2 s}\left|n_{1}^{\prime}\right|^{-2 s}\left|n^{\prime}-n_{1}^{\prime}\right|^{-2 s}\left|\hat{W}\left(n^{\prime}\right)\right|^{2}}{\left(\left\langle\sigma\left(\tau, n^{\prime}\right)\right\rangle+\left|n^{\prime}\right|^{2+2 s}\left|n_{1}^{\prime}\right|^{-2 s}\left|n^{\prime}-n_{1}^{\prime}\right|^{-2 s}\right)^{2}} \\
\leq \sup _{n}\left(\sum_{\left|n^{\prime}\right| \sim 2^{n}}\left|n^{\prime}\right|^{2+2 s}\left|n_{1}^{\prime}\right|^{-2 s}\left|n^{\prime}-n_{1}^{\prime}\right|^{-2 s}\left|\hat{W}\left(n^{\prime}\right)\right|^{2}\right. \\
\left.\quad \times \int_{\mathbb{R}} \frac{d \tau}{\left(\left\langle\sigma\left(\tau, n^{\prime}\right)\right\rangle+\left|n^{\prime}\right|^{2+2 s}\left|n_{1}^{\prime}\right|^{-2 s}\left|n^{\prime}-n_{1}^{\prime}\right|^{-2 s}\right)^{2}}\right) \\
\leq C|W|_{B_{2, \infty}^{0}}^{2} \cdot
\end{aligned}
$$

This ends the proof of Proposition 3.3.

As a last, but easy, bilinear estimate, we briefly show that we can handle terms like $\partial_{x}\left(g^{2}\right)$ in $X_{1,1}^{s,-1 / 2}$ if $g$ is only in $X_{\infty, \infty}^{s+\varepsilon, 1 / 2}$ (the $\varepsilon$ loss of regularity seems to be necessary here). Our motivation to treat such terms arises from the fact that the stochastic convolution which was studied in Proposition 2.1 belongs to such spaces (or even to $X_{1, \infty}^{s+\varepsilon, 1 / 2}$ ) if sufficient regularity is assumed on the operator $\phi$, but never belongs to $X_{1,1}^{s, 1 / 2}$, due to the lack of regularity of the Brownian motion.

Proposition 3.4. Let $-1 / 2 \leq s \leq 0$, and $\varepsilon>0$; then there is a constant $C>0$ such that for any $g \in X_{\infty, \infty}^{s+\varepsilon, 1 / 2}$,

$$
\left|\partial_{x}\left(g^{2}\right)\right|_{X_{1,1}^{s,-1 / 2}} \leq C|g|_{X_{\infty, \infty}^{s+\varepsilon, 1 / 2}}^{2} .
$$

If moreover, $g$ is supported in $[-2 T, 2 T]$ and $\partial_{x}\left(g^{2}\right)$ is considered in $X_{1,1}^{s,-1 / 2, T}$, then a factor $T^{\alpha}$ can be recovered in the right hand side above, for any $\alpha<$ $1 / 16$.

Finally, the same estimate holds if in the left hand side, $X_{1,1}^{s,-1 / 2}$ (resp. $X_{1,1}^{s,-1 / 2, T}$ ) is replaced by $Y_{s}$ (resp. $\left.Y_{s, T}\right)$.

Proof. Here again, we only sketch the proof, since the arguments are the same as in the easiest cases of the proof of Proposition 3.1, that is when some small power of $\langle\sigma\rangle$ or $\left\langle\sigma_{1}\right\rangle$ can be lost. 
Indeed, taking $f, g \in X_{\infty, \infty}^{s+\varepsilon, 1 / 2}, h \in X_{\infty, \infty}^{-s, 1 / 2}$ and setting as before $\hat{F}=$ $n^{\prime s+\varepsilon}\langle\sigma\rangle^{1 / 2} \hat{f}, \hat{G}=n^{\prime s+\varepsilon}\langle\sigma\rangle^{1 / 2} \hat{g}$ and $\hat{H}=n^{\prime-s}\langle\sigma\rangle^{1 / 2} \hat{h}$, we need to show that

$$
\begin{aligned}
& \sum_{n^{\prime} \neq 0} \sum_{\substack{n_{1}^{\prime} \neq 0 \\
n_{1}^{\prime} \neq n^{\prime}}} \int_{\tau \in \mathbb{R}} \int_{\tau_{1} \in \mathbb{R}} \frac{\left|n^{\prime}\right|^{1+s}\left|n_{1}^{\prime}\right|^{-s-\varepsilon}\left|n^{\prime}-n_{1}^{\prime}\right|^{-s-\varepsilon}}{\langle\sigma\rangle^{1 / 2}\left\langle\sigma_{1}\right\rangle^{1 / 2}\left\langle\sigma_{2}\right\rangle^{1 / 2}}\left|\hat{H}_{\tau, n^{\prime}}\right|\left|\hat{G}_{\tau_{1}, n_{1}^{\prime}}\right|\left|\hat{F}_{\tau-\tau_{1}, n^{\prime}-n_{1}^{\prime}}\right| d \tau d \tau_{1} \\
& \leq C|H|_{X_{\infty, \infty}^{0,0}}|F|_{X_{\infty, \infty}^{0,0}}|G|_{X_{\infty, \infty}^{0,0} .} .
\end{aligned}
$$

Consider e.g. Region I, where $\left\langle\sigma_{1}\right\rangle$ dominates, and where we have the inequality

$$
\left|n^{\prime}\right|^{1+s}\left|n_{1}^{\prime}\right|^{-s}\left|n^{\prime}-n_{1}^{\prime}\right|^{-s} \leq C\left\langle\sigma_{1}\right\rangle^{1 / 2}
$$

so that we are lead to estimate

$$
\sum_{n^{\prime} \neq 0} \sum_{\substack{n_{1}^{\prime} \neq 0 \\ n_{1}^{\prime} \neq n^{\prime}}} \int_{\tau \in \mathbb{R}} \int_{\tau_{1} \in \mathbb{R}} \frac{\left|n_{1}^{\prime}\right|^{-\varepsilon}\left|n^{\prime}-n_{1}^{\prime}\right|^{-\varepsilon}}{\langle\sigma\rangle^{1 / 2}\left\langle\sigma_{2}\right\rangle^{1 / 2}}\left|\hat{H}_{\tau, n^{\prime}}\right|\left|\hat{G}_{\tau_{1}, n_{1}^{\prime}}\right|\left|\hat{F}_{\tau-\tau_{1}, n^{\prime}-n_{1}^{\prime}}\right| d \tau d \tau_{1}
$$

This latest term is then handled by the same arguments as those used in Region I-a in the proof of Proposition 3.1, keeping in addition a small power of $\left\langle\sigma_{2}\right\rangle$ to be able to sum over $k$, and hence to replace the norm $|F|_{X_{1,1}^{0,0}}$ by $|F|_{X_{\infty}^{0,0}}$ (the sum over $n$ being handled by using $\left|n^{\prime}-n_{1}^{\prime}\right|^{-\varepsilon}$ ).

All the other regions are treated in the same way, and the arguments for the other statements of Proposition 3.4 are exactly the same as those of Propositions 3.2 and 3.3.

\section{Proof of Theorem 1.1 And Theorem 1.2}

As was pointed out in the introduction, it mainly remains to show that we may gain one degree of regularity in time when passing from $\partial_{x}(g f)$ to $\int_{0}^{t} U(t-s) \partial_{x}(f g)(s) d s$. The result is stated in the next proposition.

Proposition 4.1. There is a constant $C>0$ such that if $f \in X_{1,1}^{s,-1 / 2} \cap Y_{s}$, $s \in \mathbb{R}$, then $t \mapsto \int_{0}^{t} U(t-s) f(s) d s \in X_{1,1}^{s, 1 / 2, T}$ and

$$
\left|\int_{0}^{\cdot} U(\cdot-s) f(s) d s\right|_{X_{1,1}^{s, 1 / 2, T}} \leq C\left(|f|_{X_{1,1}^{s,-1 / 2}}+|f|_{Y_{s}}\right)
$$

for any $T \leq 1$.

Moreover, for any $f \in Y_{s}$, the map $t \mapsto \int_{0}^{t} U(t-s) f(s) d s$ is continuous with values in $B_{2,1}^{s}(\mathbb{T})$ and there is a constant $C>0$ such that

$$
\sup _{t \in[-T, T]}\left|\int_{0}^{t} U(t-s) f(s) d s\right|_{B_{2,1}^{s}} \leq C|f|_{Y_{s}} .
$$


Proof. The arguments of the proof are similar to those in [14]. We consider a cut-off function $\psi$ with $\psi \equiv 1$ on $[0,1]$ and supp $\psi \subset[-1,2]$; it is sufficient to prove that

$$
\left|\psi \int_{0} U(\cdot-s) f(s) d s\right|_{X_{1,1}^{s, 1 / 2}} \leq C\left(|f|_{X_{1,1}^{s,-1 / 2}}+|f|_{Y_{s}}\right)
$$

We first write

$$
\begin{aligned}
& \psi(t) \int_{0}^{t} U(t-s) f(s) d s \\
& =\psi(t) \sum_{n^{\prime} \in \mathbb{Z}} \int_{\left|\tau_{1}-n^{\prime 3}\right| \leq 1} e^{i x n^{\prime}} \hat{f}\left(\tau_{1}, n^{\prime}\right) \frac{e^{i t\left(\tau_{1}-n^{\prime 3}\right)}-1}{\tau_{1}-n^{\prime 3}} e^{i t n^{\prime 3}} d \tau_{1} \\
& +\psi(t) \sum_{n^{\prime} \in \mathbb{Z}} \int_{\left|\tau_{1}-n^{\prime 3}\right| \geq 1} e^{i x n^{\prime}} \hat{f}\left(\tau_{1}, n^{\prime}\right) \frac{e^{i t \tau_{1}}-e^{i t n^{\prime 3}}}{\tau_{1}-n^{\prime 3}} d \tau_{1} \\
& =g_{1}(t, x)+g_{2}(t, x) .
\end{aligned}
$$

To estimate $g_{1}$, we expand the exponential as

$$
\frac{e^{i t\left(\tau_{1}-n^{\prime 3}\right)}-1}{\tau_{1}-n^{\prime 3}}=\sum_{k=1}^{\infty} \frac{i^{k} t^{k}\left(\tau_{1}-n^{\prime 3}\right)^{k}}{k !}
$$

so that

$$
g_{1}(t, x)=\sum_{k=1}^{\infty} \frac{i^{k} t^{k}}{k !} \psi(t) \sum_{n^{\prime} \in \mathbb{Z}} \int_{\left|\tau_{1}-n^{\prime 3}\right| \leq 1} e^{i x n^{\prime}+i t n^{\prime 3}} \hat{f}\left(\tau_{1}, n^{\prime}\right)\left(\tau_{1}-n^{\prime 3}\right)^{k} d \tau_{1} .
$$

Let $\varphi_{k}(t)=t^{k} \psi(t) ;$ then

$$
\hat{g}_{1}\left(\tau, n^{\prime}\right)=\sum_{k=1}^{\infty} \frac{i^{k}}{k !} \int_{\left|\tau_{1}-n^{\prime 3}\right| \leq 1} \hat{\varphi}_{k}\left(\tau-n^{\prime 3}\right) \hat{f}\left(\tau_{1}, n^{\prime}\right)\left(\tau_{1}-n^{\prime 3}\right)^{k} d \tau_{1}
$$


and it follows

$$
\begin{aligned}
& \left|g_{1}\right|_{X_{1,1}^{s, 1 / 2}} \\
& =\sum_{n \in \mathbb{N}} 2^{s n} \sum_{\ell=0}^{\infty}\left(\sum_{\left|n^{\prime}\right| \sim 2^{n}} \int_{|\tau| \sim 2^{\ell}}\left|\left\langle\tau-n^{\prime 3}\right\rangle^{1 / 2} \hat{g}_{1}\left(\tau, n^{\prime}\right)\right|^{2} d \tau\right)^{1 / 2} \\
& =\sum_{n \in \mathbb{N}} 2^{s n} \sum_{\ell=0}^{\infty}\left(\sum_{\left|n^{\prime}\right| \sim 2^{n}} \int_{|\tau| \sim 2^{\ell}}\left\langle\tau-n^{\prime 3}\right\rangle\right. \\
& \left.\quad \times\left[\int_{\left|\tau_{1}-n^{\prime 3}\right| \leq 1} \sum_{k=1}^{\infty} \frac{i^{k}}{k !} \hat{\varphi}_{k}\left(\tau-n^{\prime 3}\right) \hat{f}\left(\tau_{1}, n^{\prime}\right)\left(\tau_{1}-n^{\prime 3}\right)^{k} d \tau_{1}\right]^{2} d \tau\right)^{1 / 2} .
\end{aligned}
$$

Now,

$$
\begin{aligned}
& \sum_{\left|n^{\prime}\right| \sim 2^{n}} \int_{|\tau| \sim 2^{\ell}}\left\langle\tau-n^{\prime 3}\right\rangle\left[\int_{\left|\tau_{1}-n^{\prime 3}\right| \leq 1} \sum_{k=1}^{\infty} \frac{i^{k}}{k !} \hat{\varphi}_{k}\left(\tau-n^{\prime 3}\right) \hat{f}\left(\tau_{1}, n^{\prime}\right)\left(\tau_{1}-n^{\prime 3}\right)^{k} d \tau_{1}\right]^{2} d \tau \\
\leq & \sum_{\left|n^{\prime}\right| \sim 2^{n}} \int_{|\tau| \sim 2^{\ell}}\left\langle\tau-n^{\prime 3}\right\rangle\left(\sum_{k=1}^{\infty} \frac{\left|\hat{\varphi}_{k}\left(\tau-n^{\prime 3}\right)\right|}{k !}\right)^{2} d \tau\left(\int_{\left|\tau_{1}-n^{\prime 3}\right| \leq 1}\left|\hat{f}\left(\tau_{1}, n^{\prime}\right)\right|^{2} d \tau_{1}\right) \\
\leq & \sup _{\left|n^{\prime}\right| \sim 2^{n}} \int_{|\tau| \sim 2^{\ell}}\left\langle\tau-n^{\prime 3}\right\rangle\left(\sum_{k=1}^{\infty} \frac{\left|\hat{\varphi}_{k}\left(\tau-n^{\prime 3}\right)\right|}{k !}\right)^{2} d \tau\left(\sum_{\left|n^{\prime}\right| \sim 2^{n}} \int_{\left|\tau_{1}-n^{\prime 3}\right| \leq 1}\left|\hat{f}\left(\tau_{1}, n^{\prime}\right)\right|^{2} d \tau_{1}\right) .
\end{aligned}
$$

We deduce that

$$
\begin{aligned}
& \left|g_{1}\right|_{X_{1,1}^{s, 1 / 2}} \\
& \leq \sup _{n \in \mathbb{N}} \sum_{\ell=0}^{\infty}\left(\sup _{\left|n^{\prime}\right| \sim 2^{n}} \int_{|\tau| \sim 2^{\ell}}\left\langle\tau-n^{\prime 3}\right\rangle\left(\sum_{k=1}^{\infty} \frac{\left|\hat{\varphi}_{k}\left(\tau-n^{\prime 3}\right)\right|}{k !}\right)^{2} d \tau\right)^{1 / 2} \\
& \quad \times \sum_{n \in \mathbb{N}} 2^{s n}\left(\sum_{\left|n^{\prime}\right| \sim 2^{n}} \int_{\left|\tau_{1}-n^{\prime 3}\right| \leq 1}\left|\hat{f}\left(\tau_{1}, n^{\prime}\right)\right|^{2} d \tau_{1}\right)^{1 / 2} \cdot
\end{aligned}
$$

Now, we have for $\varepsilon>0$,

$$
\begin{aligned}
& \sup _{n \in \mathbb{N}} \sum_{\ell=0}^{\infty} \sup _{\left|n^{\prime}\right| \sim 2^{n}} \int_{|\tau| \sim 2^{\ell}}\left\langle\tau-n^{\prime 3}\right\rangle\left(\sum_{k=1}^{\infty} \frac{\left|\hat{\varphi}_{k}\left(\tau-n^{\prime 3}\right)\right|}{k !}\right)^{2} d \tau \\
& \leq \sup _{n \in \mathbb{N}} \sum_{\ell \in \mathbb{N}} \sup _{\left|n^{\prime}\right| \sim 2^{n}}\left(\sup _{|\tau| \sim 2^{\ell}}\left\langle\tau-n^{\prime 3}\right\rangle^{-\varepsilon}\right) \int_{|\tau| \sim 2^{\ell}}\left\langle\tau-n^{\prime 3}\right\rangle^{1+\varepsilon}\left(\sum_{k=1}^{\infty} \frac{\left|\hat{\varphi}_{k}\left(\tau-n^{\prime 3}\right)\right|}{k !}\right)^{2} d \tau \\
& \leq C\left(\sup _{n \in \mathbb{N}} \sum_{\ell \in \mathbb{N}}\left\langle 2^{\ell}-2^{3 n}\right\rangle^{-\varepsilon}\right) \sup _{n^{\prime} \in \mathbb{N}} \int_{\mathbb{R}}\left\langle\tau-n^{\prime 3}\right\rangle^{1+\varepsilon}\left(\sum_{k=1}^{\infty} \frac{\left|\hat{\varphi}_{k}\left(\tau-n^{\prime 3}\right)\right|}{k !}\right)^{2} d \tau \\
& \leq C\left|\sum_{k=1}^{\infty} \frac{\varphi_{k}}{k !}\right|_{H^{1 / 2+\varepsilon / 2}} .
\end{aligned}
$$


Hence

$$
\left|g_{1}\right|_{X_{1,1}^{s, 1 / 2}} \leq C\left|\sum_{k=1}^{\infty} \frac{\varphi_{k}}{k !}\right|_{H^{1 / 2+\varepsilon / 2}}|f|_{X_{1,1}^{s, 0}}
$$

In order to estimate the norm of $g_{2}$, we write

$$
g_{2}(t, x)=g_{2,1}(t, x)+g_{2,2}(t, x)
$$

with

$$
g_{2,1}(t, x)=\psi(t) \sum_{n^{\prime} \in \mathbb{Z}} \int_{\left|\tau_{1}-n^{\prime 3}\right| \geq 1} e^{i x n^{\prime}} \hat{f}\left(\tau_{1}, n^{\prime}\right) \frac{e^{i t \tau_{1}}}{\tau_{1}-n^{\prime 3}} d \tau_{1}
$$

and

$$
g_{2,2}(t, x)=-\psi(t) \sum_{n^{\prime} \in \mathbb{Z}} \int_{\left|\tau_{1}-n^{\prime 3}\right| \geq 1} e^{i x n^{\prime}} \hat{f}\left(\tau_{1}, n^{\prime}\right) \frac{e^{i t n^{\prime 3}}}{\tau_{1}-{n^{\prime 3}}^{3}} d \tau_{1} .
$$

We have

$$
\hat{g}_{2,1}\left(\tau, n^{\prime}\right)=\int_{\left|\tau_{1}-n^{\prime 3}\right| \geq 1} \hat{\psi}\left(\tau-\tau_{1}\right) \frac{\hat{f}\left(\tau_{1}, n^{\prime}\right)}{\tau_{1}-n^{\prime 3}} d \tau_{1},
$$

and

$$
\begin{aligned}
& \sum_{\left|n^{\prime}\right| \sim 2^{n}} \int_{|\tau| \sim 2^{k}}\left\langle\tau-n^{\prime 3}\right\rangle\left|\hat{g}_{2,1}\left(\tau, n^{\prime}\right)\right|^{2} d \tau \\
& \leq C \sum_{\left|n^{\prime}\right| \sim 2^{n}} \int_{|\tau| \sim 2^{k}}\left[\int_{\left|\tau_{1}-n^{\prime 3}\right| \geq 1}\left\langle\tau_{1}-n^{\prime 3}\right\rangle^{1 / 2}\left|\hat{\psi}\left(\tau-\tau_{1}\right)\right|\left|\frac{\hat{f}\left(\tau_{1}, n^{\prime}\right)}{\tau_{1}-n^{\prime 3}}\right| d \tau_{1}\right]^{2} d \tau \\
& +C \sum_{\left|n^{\prime}\right| \sim 2^{n}} \int_{|\tau| \sim 2^{k}}\left[\int_{\left|\tau_{1}-n^{\prime 3}\right| \geq 1}\left\langle\tau-\tau_{1}\right\rangle^{1 / 2}\left|\hat{\psi}\left(\tau-\tau_{1}\right)\right|\left|\frac{\hat{f}\left(\tau_{1}, n^{\prime}\right)}{\tau_{1}-n^{\prime 3}}\right| d \tau_{1}\right]^{2} d \tau \\
& \leq I+I I .
\end{aligned}
$$

For the term $I$, we have

$$
I \leq C \sum_{\left|n^{\prime}\right| \sim 2^{n}} \int_{|\tau| \sim 2^{k}}\left[\int_{\mathbb{R}}\left|\hat{\psi}\left(\tau_{1}\right)\right| \frac{\left|\hat{f}\left(\tau-\tau_{1}, n^{\prime}\right)\right|}{\left\langle\tau-\tau_{1}-n^{\prime 3}\right\rangle^{1 / 2}} d \tau_{1}\right]^{2} d \tau .
$$

Let $\hat{h} \in L_{\tau, n^{\prime}}^{2}$, then

$$
\begin{aligned}
& \left|\sum_{\left|n^{\prime}\right| \sim 2^{n}} \int_{|\tau| \sim 2^{k}} \hat{h}\left(\tau, n^{\prime}\right) \int_{\mathbb{R}}\right| \hat{\psi}\left(\tau_{1}\right)\left|\frac{\left|\hat{f}\left(\tau-\tau_{1}, n^{\prime}\right)\right|}{\left\langle\tau-\tau_{1}-n^{\prime 3}\right\rangle^{1 / 2}} d \tau_{1} d \tau\right| \\
& \leq \int_{\mathbb{R}}\left|\hat{\psi}\left(\tau_{1}\right)\right|\left(\sum_{\left|n^{\prime}\right| \sim 2^{n}} \int_{|\tau| \sim 2^{k}}\left|\hat{h}\left(\tau, n^{\prime}\right)\right|^{2} d \tau\right)^{1 / 2} \\
& \quad \times\left(\sum_{\left|n^{\prime}\right| \sim 2^{n}} \int_{|\tau| \sim 2^{k}} \frac{\left|\hat{f}\left(\tau-\tau_{1}, n^{\prime}\right)\right|^{2}}{\left\langle\tau-\tau_{1}-n^{\prime 3}\right\rangle} d \tau\right)^{1 / 2} d \tau_{1} .
\end{aligned}
$$


We deduce from the preceding estimate that

$$
I \leq C\left(\int_{\mathbb{R}}\left|\psi\left(\tau_{1}\right)\right|\left(\sum_{\left|n^{\prime}\right| \sim 2^{n}} \int_{\left|\tilde{\tau}+\tau_{1}\right| \sim 2^{k}} \frac{\left|\hat{f}\left(\tilde{\tau}, n^{\prime}\right)\right|^{2}}{\left\langle\tilde{\tau}-n^{\prime 3}\right\rangle} d \tilde{\tau}\right)^{1 / 2} d \tau_{1}\right)^{2} .
$$

In the same way, we can prove that

$$
I I \leq C\left(\int_{\mathbb{R}}\left\langle\tau_{1}\right\rangle^{1 / 2}\left|\psi\left(\tau_{1}\right)\right|\left(\sum_{\left|n^{\prime}\right| \sim 2^{n}} \int_{\left|\tilde{\tau}+\tau_{1}\right| \sim 2^{k}} \frac{\left|\hat{f}\left(\tilde{\tau}, n^{\prime}\right)\right|^{2}}{\left\langle\tilde{\tau}-n^{\prime 3}\right\rangle^{2}} d \tilde{\tau}\right)^{1 / 2} d \tau_{1}\right)^{2} .
$$

Hence we have

$$
I+I I \leq C\left(\int_{\mathbb{R}}\left\langle\tau_{1}\right\rangle^{1 / 2}\left|\psi\left(\tau_{1}\right)\right|\left(\sum_{\left|n^{\prime}\right| \sim 2^{n}} \int_{\left|\tilde{\tau}+\tau_{1}\right| \sim 2^{k}} \frac{\left|\hat{f}\left(\tilde{\tau}, n^{\prime}\right)\right|^{2}}{\left\langle\tilde{\tau}-n^{\prime 3}\right\rangle} d \tilde{\tau}\right)^{1 / 2} d \tau_{1}\right)^{2}
$$

and we deduce that

$$
\begin{aligned}
& \sum_{k=0}^{\infty}\left(\sum_{\left|n^{\prime}\right| \sim 2^{n}} \int_{|\tau| \sim 2^{k}}\left\langle\tau-n^{\prime 3}\right\rangle\left|\hat{g}_{2,1}\left(\tau, n^{\prime}\right)\right|^{2} d \tau\right)^{1 / 2} \\
& \leq C \sum_{k=0}^{\infty} \int_{\mathbb{R}}\left\langle\tau_{1}\right\rangle^{1 / 2}\left|\hat{\psi}\left(\tau_{1}\right)\right|\left(\sum_{\left|n^{\prime}\right| \sim 2^{n}} \int_{\left|\tilde{\tau}+\tau_{1}\right| \sim 2^{k}} \frac{\left|\hat{f}\left(\tilde{\tau}, n^{\prime}\right)\right|^{2}}{\left\langle\tilde{\tau}-n^{\prime 3}\right\rangle} d \tilde{\tau}\right)^{1 / 2} d \tau_{1} \\
& \leq C \sum_{k_{1}=0}^{\infty} \int_{\left|\tau_{1}\right| \sim 2^{k_{1}}}\left\langle\tau_{1}\right\rangle^{1 / 2}\left|\hat{\psi}\left(\tau_{1}\right)\right| \\
& \quad \times\left(\sum_{k<k_{1}-4}+\sum_{k_{1}-4 \leq k \leq k_{1}+4}+\sum_{k>k_{1}+4}\right)\left(\sum_{\left|n^{\prime}\right| \sim 2^{n}} \int_{\left|\tilde{\tau}+\tau_{1}\right| \sim 2^{k}} \frac{\left|\hat{f}\left(\tilde{\tau}, n^{\prime}\right)\right|^{2}}{\left\langle\tilde{\tau}-n^{\prime 3}\right\rangle} d \tilde{\tau}\right)^{1 / 2} d \tau_{1} .
\end{aligned}
$$

Since

$$
\begin{aligned}
& \left(\sum_{k<k_{1}-4}+\sum_{k_{1}-4 \leq k \leq k_{1}+4}+\sum_{k>k_{1}+4}\right)\left(\sum_{\left|n^{\prime}\right| \sim 2^{n}} \int_{\left|\tilde{\tau}+\tau_{1}\right| \sim 2^{k}} \frac{\left|\hat{f}\left(\tilde{\tau}, n^{\prime}\right)\right|^{2}}{\left\langle\tilde{\tau}-n^{\prime 3}\right\rangle} d \tilde{\tau}\right)^{1 / 2} \\
& \leq C\left(k_{1}-4\right)\left(\sum_{\left|n^{\prime}\right| \sim 2^{n}} \sum_{j=-1}^{+1} \int_{|\tilde{\tau}| \sim 2^{k_{1}+j}} \frac{\left|\hat{f}\left(\tilde{\tau}, n^{\prime}\right)\right|^{2}}{\left\langle\tilde{\tau}-n^{\prime 3}\right\rangle} d \tilde{\tau}\right)^{1 / 2} \\
& +8\left(\sum_{\left|n^{\prime}\right| \sim 2^{n}} \int_{|\tilde{\tau}| \sim 2^{k_{1}+5}} \frac{\left|\hat{f}\left(\tilde{\tau}, n^{\prime}\right)\right|^{2}}{\left\langle\tilde{\tau}-n^{\prime 3}\right\rangle} d \tilde{\tau}\right)^{1 / 2} \\
& +C \sum_{k \in \mathbb{N}}\left(\sum_{\left|n^{\prime}\right| \sim 2^{n}} \int_{|\tilde{\tau}| \sim 2^{k}} \frac{\left|\hat{f}\left(\tilde{\tau}, n^{\prime}\right)\right|^{2}}{\left\langle\tilde{\tau}-n^{\prime 3}\right\rangle} d \tilde{\tau}\right)^{1 / 2},
\end{aligned}
$$


we may easily bound the preceding term by

$$
\begin{aligned}
& C \sum_{k_{1}=0}^{\infty} \int_{\left|\tau_{1}\right| \sim 2^{k_{1}}}\left(1+k_{1}\right) 2^{k_{1} / 2}\left|\hat{\psi}\left(\tau_{1}\right)\right| d \tau_{1} \sum_{k=0}^{\infty}\left(\sum_{\left|n^{\prime}\right| \sim 2^{n}} \int_{|\tau| \sim 2^{k}} \frac{\left|\hat{f}\left(\tilde{\tau}, n^{\prime}\right)\right|^{2}}{\left\langle\tilde{\tau}-n^{\prime 3}\right\rangle} d \tilde{\tau}\right)^{1 / 2} \\
& \leq C_{\varepsilon}\left|\langle\tau\rangle^{1 / 2+\varepsilon} \hat{\psi}\right|_{L^{1}(\mathbb{R})} \sum_{k=0}^{\infty}\left(\sum_{\left|n^{\prime}\right| \sim 2^{n}} \int_{|\tau| \sim 2^{k}} \frac{\left|\hat{f}\left(\tilde{\tau}, n^{\prime}\right)\right|^{2}}{\left\langle\tilde{\tau}-n^{\prime 3}\right\rangle} d \tilde{\tau}\right)^{1 / 2}
\end{aligned}
$$

thus

$$
\left|g_{2,1}\right|_{X_{1,1}^{s, 1 / 2}} \leq C_{\varepsilon}\left|\langle\tau\rangle^{1 / 2+\varepsilon} \hat{\psi}\right|_{L^{1}(\mathbb{R})}|f|_{X_{1,1}^{s,-1 / 2}}
$$

At last,

$$
\hat{g}_{2,2}\left(\tau, n^{\prime}\right)=\hat{\psi}\left(\tau-n^{\prime 3}\right) \int_{\left|\tau_{1}-n^{\prime 3}\right| \geq 1} \frac{\hat{f}\left(\tau_{1}, n^{\prime}\right)}{\tau_{1}-n^{\prime 3}} d \tau_{1}
$$

and hence

$$
\begin{aligned}
& \sum_{n \in \mathbb{N}} 2^{s n} \sum_{k \in \mathbb{N}}\left(\int_{|\tau| \sim 2^{k}} \sum_{\left|n^{\prime}\right| 2^{n}}\left\langle\tau-n^{\prime 3}\right\rangle\left|\hat{g}_{2,2}\left(\tau, n^{\prime}\right)\right|^{2} d \tau\right)^{1 / 2} \\
& \leq \sum_{n \in \mathbb{N}} 2^{s n} \sum_{k \in \mathbb{N}}\left(\sup _{\left|n^{\prime}\right| \sim 2^{n}} \int_{|\tau| \sim 2^{k}}\left\langle\tau-n^{\prime 3}\right\rangle\left|\hat{\psi}\left(\tau-n^{\prime 3}\right)\right|^{2} d \tau\right)^{1 / 2} \\
& \quad \times\left(\sum_{\left|n^{\prime}\right| \sim 2^{n}}\left(\int_{\mathbb{R}} \frac{\left|\hat{f}\left(\tau_{1}, n^{\prime}\right)\right|}{\left\langle\tau_{1}-n^{\prime 3}\right\rangle} d \tau_{1}\right)^{2}\right)^{1 / 2} \\
& \leq C \\
& \quad \sum_{n \in \mathbb{N}} 2^{s n}\left(\sum_{k \in \mathbb{N}}\left\langle 2^{k}-2^{3 n}\right\rangle^{-\varepsilon}\right) \\
& \quad \times\left(\sup _{\left|n^{\prime}\right| \sim 2^{n}} \int_{\mathbb{R}}\left\langle\tau-n^{\prime 3}\right\rangle^{1+\varepsilon}\left|\hat{\psi}\left(\tau-n^{\prime 3}\right)\right|^{2} d \tau\right)^{1 / 2} \\
& \quad \times\left(\sum_{\left|n^{\prime}\right| \sim 2^{n}}\left(\int_{\mathbb{R}} \frac{\left|\hat{f}\left(\tau_{1}, n^{\prime}\right)\right|}{\left\langle\tau_{1}-n^{\prime 3}\right\rangle} d \tau_{1}\right)^{2}\right)^{1 / 2} \\
& \leq C|\psi|_{H^{1 / 2+\varepsilon / 2}|f|_{Y_{s}} .}
\end{aligned}
$$

This ends the proof of the first estimate in Proposition 4.1. The proof of continuity with values in $B_{2,1}^{s}$ and the second estimate follow in an obvious way from a slight modification of the proof of Lemma 2.2 in [10].

The next lemma shows that the free term in equation (1.5) belongs to $X_{1,1}^{\sigma, 1 / 2, T}$ if $u_{0}$ is in $B_{2,1}^{\sigma}(\mathbb{T})$. 
Lemma 4.1. Let $u_{0} \in B_{2,1}^{\sigma}(\mathbb{T})$, and $T \leq 1$. Then $U(t) u_{0} \in X_{1,1}^{\sigma, 1 / 2, T}$ and there is a constant $C>0$ such that

$$
\left|U(t) u_{0}\right|_{X_{1,1}^{\sigma, 1 / 2, T}} \leq C\left|u_{0}\right|_{B_{2,1}^{\sigma}} .
$$

Proof. Let $\psi$ be a cut-off function with $\psi \equiv 1$ on $[0,1]$ and let us prove that $\left|\psi U(t) u_{0}\right|_{X_{1,1}^{\sigma, 1 / 2}} \leq C\left|u_{0}\right|_{B_{2,1}^{\sigma}}$.

We use the fact that $X_{1, \infty}^{\sigma, 1 / 2+\varepsilon} \subset X_{1,1}^{\sigma, 1 / 2}$ for any $\varepsilon>0$, and that

$$
\widehat{\psi U(t) u_{0}}\left(\tau, n^{\prime}\right)=\hat{u}_{0}\left(n^{\prime}\right) \psi\left(\tau-n^{\prime 3}\right)
$$

to get the following bound :

$$
\begin{aligned}
& \left|\psi U(t) u_{0}\right|_{X_{1,1}^{\sigma, 1 / 2}} \leq C_{\varepsilon}\left|\psi U(t) u_{0}\right|_{X_{1, \infty}^{\sigma, 1 / 2+\varepsilon}} \\
& \leq C_{\varepsilon} \sum_{n=0}^{\infty} 2^{\sigma n} \sup _{k \geq 0}\left(\sum_{\left|n^{\prime}\right| \sim 2^{n}} \int_{|\tau| \sim 2^{k}}\left\langle\tau-n^{\prime 3}\right\rangle^{1+2 \varepsilon}\left|\hat{u}_{0}\left(n^{\prime}\right)\right|^{2}\left|\hat{\psi}\left(\tau-n^{\prime 3}\right)\right|^{2} d \tau\right)^{1 / 2} \\
& \leq C_{\varepsilon} \sum_{n=0}^{\infty} 2^{\sigma n} \sup _{k \geq 0}\left(\sum_{\left|n^{\prime}\right| \sim 2^{n}} \sum_{j=0}^{\infty} \int_{\substack{|\tau| \sim 2^{k} \\
\left|\tau-n^{\prime 3}\right| \sim 2^{j}}}\left\langle\tau-n^{\prime 3}\right\rangle^{1+2 \varepsilon}\left|\hat{u}_{0}\left(n^{\prime}\right)\right|^{2}\left|\hat{\psi}\left(\tau-n^{\prime 3}\right)\right|^{2} d \tau\right)^{1 / 2} \\
& \leq C_{\varepsilon} \sum_{n=0}^{\infty} 2^{\sigma n} \sum_{j=0}^{\infty}\left(\sum_{\left|n^{\prime}\right| \sim 2^{n}} \int_{\left|\tau-n^{\prime 3}\right| \sim 2^{j}}\left\langle\tau-n^{\prime 3}\right\rangle^{1+2 \varepsilon}\left|\hat{u}_{0}\left(n^{\prime}\right)\right|^{2}\left|\hat{\psi}\left(\tau-n^{\prime 3}\right)\right|^{2} d \tau\right)^{1 / 2} \\
& \leq C_{\varepsilon} \sum_{n=0}^{\infty} 2^{\sigma n}\left(\sum_{\left|n^{\prime}\right| \sim 2^{n}}\left|\hat{u}_{0}\left(n^{\prime}\right)\right|^{2}\right)^{1 / 2} \sum_{j=0}^{\infty}\left(\int_{|\tau| \sim 2^{j}}\langle\tau\rangle^{1+2 \varepsilon}|\hat{\psi}(\tau)|^{2} d \tau\right)^{1 / 2} \\
& \leq C_{\varepsilon}\left|u_{0}\right|_{B_{2,1}^{\sigma}}|\psi|_{B_{2,1}^{1 / 2+\varepsilon}} .
\end{aligned}
$$

Proof of Theorem 1.1. We now have all the estimates in hand, and we proceed exactly as in [3]; we work path-wise on equation (1.5), using a fixed point argument in the space $X_{1,1}^{\sigma, 1 / 2, T}$ with $-1 / 2 \leq \sigma<s, s$ being defined by the assumption on $\phi$, and $T \leq 1$ sufficiently small.

Let $u_{0} \mathcal{F}_{0}$-measurable with $u_{0} \in B_{2,1}^{\sigma}(\mathbb{T})$ almost surely, $\sigma$ as above and assume first that $\hat{u}_{0}(0)=0$ a.s. We set

$$
z(t)=U(t) u_{0}
$$

then by Lemma 4.1, $z \in X_{1,1}^{\sigma, 1 / 2, T}$ for any $T \leq 1$, a.s. and

$$
|z|_{X_{1,1}^{\sigma, 1 / 2, T}} \leq C\left|u_{0}\right|_{B_{2,1}^{\sigma}} \quad \text { a.s. }
$$


Let $w(t)$ be defined by (1.4). By Proposition 2.1, $w \in X_{1, \infty}^{\sigma^{\prime}, 1 / 2, T} \subset X_{\infty, \infty}^{\sigma^{\prime}, 1 / 2, T}$ almost surely, for any $\sigma^{\prime}$ with $\sigma<\sigma^{\prime}<s$. We fix such a $\sigma^{\prime}$ and consider $\omega \in \Omega$ such that $u_{0} \in B_{2,1}^{\sigma}(\mathbb{T})$ and $w \in X_{1, \infty}^{\sigma^{\prime}, 1 / 2, T}$ for any $T \leq 1$, a.s.

In terms of $v(t)=u(t)-z(t)-w(t)$, equation (1.5) is written as

$$
v(t)=\mathcal{T} v(t):=-\frac{1}{2} \int_{0}^{t} U(t-s) \partial_{x}\left(v^{2}+w^{2}+z^{2}+2 v w+2 v z+2 w z\right)(s) d s
$$

Taking $0<\alpha<1 / 16$ in Propositions 3.2, 3.3 and 3.4, and $\varepsilon \leq \alpha / 2$ in Proposition 4.1 , we easily get the existence of a constant $C_{\alpha}>0$ such that

$$
|\mathcal{T} v|_{X_{1,1}^{\sigma, 1 / 2, T}} \leq C_{\alpha} T^{\alpha / 2}\left(|v|_{X_{1,1}^{\sigma, 1 / 2, T}}^{2}+|w|_{X_{1, \infty}^{\sigma, 1 / 2, T}}^{2}+\left|u_{0}\right|_{B_{2,1}^{\sigma}}^{2}\right)
$$

In the same way, if $v_{1}, v_{2} \in X_{1,1}^{\sigma, 1 / 2, T}$ then

$$
\begin{aligned}
\left|\mathcal{T} v_{1}-\mathcal{T} v_{2}\right|_{X_{1,1}^{\sigma, 2, T}} \leq C_{\alpha} T^{\alpha / 2}\left(\left|v_{1}\right|_{X_{1,1}^{\sigma, 1 / 2, T}}+\left|v_{2}\right|_{X_{1,1}^{\sigma, 1 / 2, T}}\right. & \\
& \left.+|w|_{X_{1, \infty}^{\sigma, 1 / 2, T}}+\left|u_{0}\right|_{B_{2,1}^{\sigma}}\right)\left|v_{1}-v_{2}\right|_{X_{1,1}^{\sigma, 1 / 2, T}}
\end{aligned}
$$

Hence, setting first

$$
R_{\omega}^{t}=|w|_{X_{1, \infty}^{\sigma, 1 / 2, t}}+\left|u_{0}\right|_{B_{2,1}^{\sigma}}
$$

and then defining the stopping time $T_{\omega}$ by

$$
T_{\omega}=\inf \left\{t>0, \quad 2 C_{\alpha} t^{\alpha / 2} R_{\omega}^{t} \geq 1 / 2\right\}
$$

it is easily checked that $\mathcal{T}$ maps the ball of radius $R_{\omega}^{T_{\omega}}$ in $X_{1,1}^{\sigma, 1 / 2, T_{\omega}}$ into itself, and that

$$
\left|\mathcal{T} v_{1}-\mathcal{T} v_{2}\right|_{X_{1,1}^{\sigma, 1 / 2, T_{\omega}}} \leq \frac{3}{4}\left|v_{1}-v_{2}\right|_{X_{1,1}^{\sigma, 1 / 2, T_{\omega}}}
$$

Hence $\mathcal{T}$ has a unique fixed point, which is the unique solution of (4.3) in $X_{1,1}^{\sigma, 1 / 2, T_{\omega}}$.

It follows from classical arguments and the second part of Proposition 4.1 that $z$ and $v$ are in $C\left(\left[0, T_{\omega}\right] ; B_{2,1}^{\sigma}(\mathbb{T})\right)$ a.s. On the other hand, since $\phi \in$ $L_{2}^{0, s}$ and $U(t)$ is a unitary group in $H^{s}(\mathbb{T})$, we have $w \in C\left(\left[0, T_{\omega}\right] ; H^{s}(\mathbb{T})\right) \subset$ $C\left(\left[0, T_{\omega}\right] ; B_{2,1}^{\sigma}(\mathbb{T})\right)$ by Theorem 6.10 in [8]. Hence, the solution $u=v+z+w$ of $(1.5)$ is almost surely continuous with values in $B_{2,1}^{\sigma}(\mathbb{T})$.

One classically get rid of the condition $\hat{u}_{0}(0)=0$ a.s. by considering $v(t, x)=$ $u\left(t, x+\alpha_{0} t\right)-\alpha_{0}$ with $\alpha_{0}=\int_{\mathbb{T}} u_{0}(x) d x$; indeed, $v$ then satisfies the KdV equation (1.2) and the condition $\hat{v}_{0}(0)=0$.

This ends the proof of Theorem 1.1.

We now explain how we can get rid of the condition that the spatial mean of the noise is zero almost surely at any time. 
Proposition 4.2. The conclusion of Theorem 1.1 is still true without the assumption that $\operatorname{Im} \phi \subset \operatorname{span}\left\{e_{j}, j \geq 1\right\}$.

Proof. Let $P$ be the orthogonal projector on span $\left\{e_{0}\right\}$ in $L^{2}(\mathbb{T})$ i.e. $(P u)(x)=$ $\left(u, e_{0}\right) e_{0}$ for $u \in L^{2}(\mathbb{T})$, where $(\cdot, \cdot)$ denotes the inner product in $L^{2}(\mathbb{T})$. Then, clearly, $\tilde{\phi}=(I-P) \phi$ satisfies $\operatorname{Im} \tilde{\phi} \subset \operatorname{span}\left\{e_{j}, j \geq 1\right\}$; on the other hand, $W=P \phi \tilde{W}+\tilde{\phi} \tilde{W}$, and $\tilde{\beta}(t)=P \phi \tilde{W}(t)=\sum_{k \in \mathbb{N}}\left(\phi e_{k}, e_{0}\right) \beta_{k}(t) e_{0}$ is a real valued Brownian motion since $\sum_{k \in \mathbb{N}}\left(\phi e_{k}, e_{0}\right)^{2}=\left|\phi^{*} e_{0}\right|_{L^{2}(\mathbb{T})}^{2}<+\infty$.

Let $v=u-\tilde{\beta}$, then if $u$ satisfies the $\mathrm{KdV}$ equation (1.2), $v$ satisfies

$$
\left\{\begin{array}{l}
d v+\left(\partial_{x}^{3} v+(v+\tilde{\beta}) \partial_{x} v\right) d t=\tilde{\phi} d \tilde{W} \\
v(0)=u_{0}
\end{array}\right.
$$

and setting $\tilde{v}(t, x)=v\left(t, x+\int_{0}^{t} \tilde{\beta}(s) d s\right)$, we get the equation for $\tilde{v}$

$$
\left\{\begin{array}{l}
d \tilde{v}+\left(\partial_{x}^{3} \tilde{v}+\tilde{v} \partial_{x} \tilde{v}\right) d t=d \hat{W} \\
\tilde{v}(0)=u_{0}
\end{array}\right.
$$

with $\hat{W}(t, x)=\sum_{k \in \mathbb{N}}\left(\tilde{\phi} e_{k}\right)\left(x-\int_{0}^{t} \tilde{\beta}(s) d s\right) \beta_{k}(t)$ and it is clear that we can apply all the arguments of the proof of Theorem 1.1 to equation (4.4), leading to the existence and uniqueness of $\tilde{v}$ from which we deduce the existence and uniqueness of $u$. Indeed, note that in Proposition 2.1, $\phi$ was allowed to depend on $t$ and $\omega$ provided that it was in $L^{\infty}\left((0, T) \times \Omega ; L_{2}^{0, s}\right)$, which is obviously the case here.

Proof of Theorem 1.2. The arguments are exactly the same as in [3]: let $T>0$ fixed; under the assumptions of Theorem 1.2, considering a sequence $\phi_{n}$ in $L_{2}^{0,4}$ such that $\phi_{n} \rightarrow \phi$ in $L_{2}^{0,0}$ and a sequence $u_{0, n}$ in $L^{2}\left(\Omega ; H^{3}(\mathbb{T})\right)$ such that $u_{0, n} \rightarrow u_{0}$ in $L^{2}\left(\Omega ; L^{2}(\mathbb{T})\right)$; one can easily prove (see [2]) the existence of a unique solution $u_{n}$ in $C\left([0, T] ; H^{3}(\mathbb{T})\right)$ of

$$
u_{n}(t)=U(t) u_{0, n}-\frac{1}{2} \int_{0}^{t} U(t-s) \partial_{x}\left(u_{n}^{2}(s)\right) d s+\int_{0}^{t} U(t-s) \phi_{n} d \tilde{W}(s) .
$$

Using Itô formula on $\left|u_{n}\right|_{L^{2}(\mathbb{T})}^{2}$ and a martingale inequality, one gets as in [3]

$$
\mathbb{E}\left(\sup _{t \in[0, T]}\left|u_{n}(t)\right|_{L^{2}(\mathbb{T})}^{2}\right) \leq \mathbb{E}\left(\left|u_{0, n}\right|_{L^{2}(\mathbb{T})}^{2}\right)+C(T)\left\|\phi_{n}\right\|_{L_{2}^{0,0}}^{2}
$$

hence, up to a subsequence, $u_{n}$ converges in $L^{2}\left(\Omega ; L^{\infty}\left(0, T ; L^{2}(\mathbb{T})\right)\right)$ weak star to some process $\tilde{u}$. Then if $\mathcal{T}_{n}$ is defined in the same way as $\mathcal{T}$ in the proof of Theorem 2.1, replacing $u_{0}$ and $\phi$ respectively by $u_{0, n}$ and $\phi_{n}$, one shows 
that, given $\sigma<0, \mathcal{T}_{n}$ is a uniform contraction in the ball of radius $R_{\omega}^{T_{\omega}}$ in $X_{1,1}^{\sigma, 1 / 2, T_{\omega}}$; moreover the unique fixed point of $\mathcal{T}_{n}$ is equal to $u_{n}$, which, as a result, converges to $u$ (the solution given by Theorem 1.1) in $X_{1,1}^{\sigma, 1 / 2, T_{\omega}}$ for any $\sigma<0$. It follows that $u=\tilde{u}$ a.s. on $\left[0, T_{\omega}\right]$, and that

$$
\left|u\left(T_{\omega}\right)\right|_{B_{2,1}^{\sigma}(\mathbb{T})} \leq C_{\sigma}\left|u\left(T_{\omega}\right)\right|_{L^{2}(\mathbb{T})} \leq|\tilde{u}|_{L^{\infty}\left(0, T ; L^{2}(\mathbb{T})\right)} \quad \text { a.s. }
$$

so that $u$ may be extended to $[0, T]$ almost surely, giving the result.

Acknowledgments. The authors would like to thank Professor Masayoshi Takeda for mentioning the regularity in Besov spaces of Brownian motion to them.

\section{REFERENCES}

[1] J. Bona, R. Smith, The initial value problem for the Korteweg-de Vries equation, Phil. Trans. Roy. Soc. London, A 278, (1975), p. 555-601.

[2] A. de Bouard, A. Debussche, On the stochastic Korteweg-de Vries equation, J. Funct. Anal. 154, (1998), p. 215-251.

[3] A. de Bouard, A. Debussche, Y. Tsutsumi, White noise driven Korteweg-de Vries equation, J. Funct. Anal. 169, (1999), p. 532-558.

[4] J. Bourgain, Fourier restriction phenomena for certain lattice subsets and applications to nonlinear evolution equations, part II, Geom. Funct. Anal. 3, (1993), p. 209-262.

[5] H. Y. Chang, Ch. Lien, S. Sukarto, S. Raychaudhury, J. Hill, E. K. Tsikis and K. E. Lonngren, Propagation of Ion-Acoustic solitons in a non-quiescent plasma, Plasma Phys. Controlled Fusion 28, (1986), p. 675-681.

[6] Z. Ciesielski, Orlicz spaces, spline systems, and Brownian motion, Constr. Approx. 9, (1993), p. 191-208.

[7] J. Colliander, M. Keel, G. Staffilani, H. Takaoka, T. Tao, Sharp global well-posedness for $K d V$ and modified $K d V$ on $\mathbb{R}$ and $\mathbb{T}$, J. Amer. Math. Soc. 16, (2003), p. 705-749.

[8] G. Da Prato, J. Zabczyk, Stochastic equations in infinite dimensions, in "Encyclopedia of Mathematics and its Application", Cambridge University Press, Cambridge, 1992.

[9] J. Ginibre, Le problème de Cauchy pour des EDP semi-linéaires périodiques en variables d'espace (d'après Bourgain), Séminaire Bourbaki no 796, Astérisque 237, (1996), p. 163-187.

[10] J. Ginibre, Y. Tsutsumi, G. Velo, The Cauchy problem for the Zakharov system, J. Funct. Anal. 151, (1997), p. 384-436.

[11] O. Goubet, Asymptotic smoothing effect for weakly damped forced Korteweg-de Vries equations, Disc. Cont. Dynamical Syst. 6, (2000), p. 625-644.

[12] R. Herman, The stochastic, damped Korteweg-de Vries equation, J. Phys. A. 23 (1990), p. 1063-1084.

[13] C.E. Kenig, G. Ponce and L. Vega, Well-posedness of the initial value problem for the Korteweg-de Vries equation, J. Amer. Math. Soc. 4, (1991), p. 323-347.

[14] C.E. Kenig, G. Ponce and L. Vega, The Cauchy problem for the Korteweg-de Vries equation in Sobolev spaces of negative indices, Duke Math. J. 71, (1993), p. 1-21.

[15] C.E. Kenig, G. Ponce and L. Vega, A bilinear estimate with application to the $K d V$ equation, J. Amer. Math. Soc. 9, (1996), p. 573-604. 
[16] V. V. Konotop and L. Vasquez, Nonlinear random waves, World Scientific, Singapore, 1994.

[17] B. Roynette, Mouvement brownien et espaces de Besov, Stochastics Stochastics Rep. 43, (1993), p. 221-260.

[18] J.C. Saut, R.Temam, Remarks on the Korteweg-de Vries equation, Israel J. Math. 24, (1976), p. 78-87.

[19] M. Scalerandi, A. Romano and C. A. Condat, Korteweg-de Vries solitons under additive stochastic perturbations, Phys. Review E 58, (1998), p. 4166-4173.

[20] N. Tzvetkov, Remarque sur la régularité locale de l'équation de Kadomtsev-PetviashviliII, C. R. Acad. Sci. Paris, 326, (1998), p. 709-712.

[21] M. Wadati, Stochastic Korteweg-de Vries equation, J. Phys. Soc. Japan 52 (1983), p. 2642-2648.

[22] M. Wadati, Y. Akutsu, Stochastic Korteweg-de Vries Equation with and without Damping, J. Phys. Soc. Japan 53 (1984), p. 3342. 